\title{
Generalized Drinfel'd-Sokolov Hierarchies
}

\author{
Mark F. de Groot ${ }^{1}$, Timothy J. Hollowood ${ }^{2}$ and J. Luis Miramontes ${ }^{2}$ \\ ${ }^{1}$ Institute for Advanced Study, Olden Lane, Princeton, NJ 08540, USA \\ 2 Joseph Henry Laboratories, Department of Physics, Princeton University, Princeton, NJ 08544, \\ USA
}

Received April 16, 1991; in revised form September 20, 1991

\begin{abstract}
A general approach is adopted to the construction of integrable hierarchies of partial differential equations. A series of hierarchies associated to untwisted Kac-Moody algebras, and conjugacy classes of the Weyl group of the underlying finite Lie algebra, is obtained. The generalized KdV hierarchies of V.G. Drinfel'd and V.V. Sokolov are obtained as the special case for the Coxeter element. Various examples of the general formalism are treated in some detail; including the fractional $\mathrm{KdV}$ hierarchies.
\end{abstract}

\section{Introduction}

It is only comparatively recently that some degree of mastery of non-linear equations has been achieved, and in this regard it is the notion of integrability that has proved one of the most useful concepts. Prior to the discovery of the Korteweg de Vries (KdV) and Nonlinear Schrödinger (NLS) equations, there were very few known integrable systems, examples being the harmonic oscillator and rigid body motion. (There was also the exactly solvable Ising model of Statistical Mechanics, the only such system with an infinite number of degrees of freedom.) In the 1960's the KdV equation was shown to have an infinite number of conservation laws and in fact to be integrable (see [1] for a nice discussion of the history of these and subsequent developments). The existence of solitons solutions of the KdV equation, which is a hallmark of integrable systems, is the result of an apparently delicate interplay between dispersion on the one hand and nonlinearity on the other - the latter causing a steepening of the wave-front. Despite the seemingly delicate nature of the interplay required, the $\mathrm{KdV}$ equation finds a wide application in physics. Five years after the efforts required to demonstrate the integrability of this equation, Zakharov and Shabat found the Lax pair formulation of the NLS equation, and shortly thereafter the sine-Gordon equation was similarly treated (these equations too are widespread). Subsequent to the successful treatment of the above three equation, all sorts of generalizations were obtained [1]. 
In the work of Drinfel'd and Sokolov [2] many disparate threads were pulled together in an elegant manner. What they did was to proceed roughly along the following lines. If the following definition is made

$$
L=D^{k}+\sum_{i=0}^{k-1} u_{i} D^{i}, \quad D=\frac{d}{d x}, \quad A=\sum_{i=0}^{m} v_{i} D^{i},
$$

then the scalar Lax equation is given by

$$
\frac{d L}{d t}=[A, L]
$$

where if the $u_{i}$ are given, the $v_{i}$ are determined by requiring that the evolution equation is a consistent equation. A matrix Lax pair formulation of the above can be given without much difficulty, by choosing

$$
\tilde{L}=\partial_{x}+q+\Lambda=\partial_{x}+\left(\begin{array}{cccc}
0 & \cdots & 0 & 0 \\
\vdots & & \vdots & \vdots \\
0 & \cdots & 0 & 0 \\
u_{1} & \cdots & u_{n} & 0
\end{array}\right)+\left(\begin{array}{ccc}
1 & & \\
& \ddots & \\
& & 1 \\
z & &
\end{array}\right)
$$

with $z$ a spectral parameter, in which case (1.2) becomes the matrix Lax equation:

$$
\frac{d \tilde{L}}{d t}=[\tilde{A}, \tilde{L}],
$$

where the differential operator $A$, of order $m$, becomes a matrix $\tilde{A}$ of the form $\tilde{A}=$ $\sum_{i=0}^{m} z^{i} \tilde{v}_{i}$. However, it was by no means clear to how to generalize this construction. One could for example consider a more general $q$, allowing it to be a lower triangular matrix, but then the matrix $\tilde{A}$ whose commutator with $\widetilde{L}$ would give the evolution equation of the hierarchy at a certain order, is not uniquely defined. However, introducing the following notion of gauge-equivalence

$$
\tilde{L} \rightarrow \tilde{L}^{\prime}=N \tilde{L} N^{-1}
$$

with $N$ a lower-triangular matrix with 1's on the diagonal, this indeterminacy in $\tilde{A}$ can be circumvented (in a manner which will be explained) and effectively the situation is that considered initially in Eqs. (1.1) and (1.2). The benefit, though, of proceeding in this manner is that whereas the $q$ in Eq. (1.3) has no group-theoretic interpretation, the lower-triangular $q$ can be considered elements of the Borel subalgebra, while the $N$ can be considered an exponentiated subalgebra. This interpretation allows a natural generalization, and so in fact $\mathrm{KdV}$ type equations can be defined for arbitrary Kac-Moody algebras (the actual construction required the principal and homogeneous gradations which we shall come to). Similar results were obtained in [3], though the association here was made between $\mathrm{mKdV}$ equations and untwisted affine Lie algebras. In this same work a suggestion was made concerning the proper setting in which these developments were to be viewed and it was this suggestion that provided the impetus for the present work.

Apart from the intrinsic interest of the generalizations considered here, they being in some cases new integrable systems and in others familiar systems with a 
novel interpretation, there are other motivations connected with the attempt to understand quantum gravity in two dimensions. In the one-matrix model it was shown that the partition function can be written as the square-root of the $\tau$-function, subject to an extra constraint, namely the string equation, and hence the specific heat can be considered as a potential $u=u\left(x, t_{1}, t_{2}, \ldots\right)$ which satisfies the $\mathrm{KdV}$ hierarchy of equations [4]. More general matrix-models are similarly connected with the $S L(N)-\mathrm{KdV}$ hierarchies of [2]. On the other hand it has recently been shown that twisted $N=2$ super-conformal models of the minimal $c=\frac{3 k}{(k+2)}$ series, if coupled to topological gravity, give correlation functions which obey relations similar to those encountered in $k+1$-matrix models (the minimal models are obtained by considering $\frac{S U(k+1)}{S U(k) \times U(1)}$ cosets at level one, and so $\frac{S U(2)}{U(1)}$ describes the one-matrix model which in turn corresponds to pure gravity). It is desirable to understand this connexion better and in addition to consider what happens when we replace the above cosets by the non-minimal $N=2$ coset models of [5] based on Hermitian symmetric spaces $G / H$, where $G$ is simply laced and has KacMoody level equal to one. What, for example, are the matrix models to which this corresponds?

Another motivation concerns the generalized $W$-algebras and the fractional $\mathrm{KdV}$ hierarchies. In [6] Polyakov demonstrated a relationship between $W_{N}$-algebras and a constrained $S L(N)$ current algebra. A similar structure emerges in the Drinfel'd-Sokolov picture, where the classical commutation relations of the $W_{N^{-}}$ algebra constitute one of the two Hamiltonian structures of the $S L(N)-\mathrm{KdV}$ hierarchies. In [7] generalizations of the above $W$-algebras were introduced, namely $W_{N}^{l}$-algebras, where $l$ runs from 1 to $N-1$ and the $W_{N}^{1}$-algebra is the standard $W_{N}$-algebra. In [15] fractional $\mathrm{KdV}$ hierarchies were introduced, where these now bear the same relation to the $W_{N}^{l}$-algebras as the earlier $S L(N)-\mathrm{KdV}$ equations bear to the $W_{N}$-algebras.

An outline of the paper is as follows. We restrict our attention to the untwisted algebras, which have a nice realization in terms of an underlying finite Lie algebra $g$. We introduce the notion of a gradation of a Kac-Moody algebra, that is to say a decomposition of the algebra $\hat{g}=\bigoplus_{i} \hat{g}_{i}$ such that $\left[\hat{g}_{i}, \hat{g}_{j}\right]=\hat{g}_{i+j}$. We then discuss the classification of maximally commuting subalgebras with central extension of the given Kac-Moody algebra, which are known as Heisenberg subalgebras. An important element of this classification will be the Weyl group of the finite Lie algebra $g$, generated by reflection in the hyperplanes normal to the vectors of the root-system and which is a normal subgroup of the group $\operatorname{Aut}\left(\Phi_{g}\right)$, the automorphism group of the root system of $g$. We then proceed to show how generalized hierarchies can be defined and discuss various examples, including the fractional $\mathrm{KdV}$ hierarchy mentioned above. Finally we present our conclusions.

\section{Kac-Moody Algebras}

In this section some of the formalism of Kac-Moody algebras will be introduced, but only the bare minimum necessary to understand the construction in the following 
sections. A thorough review of the theory may be found in the excellent book by $\mathrm{Kac}$ [8]. The section is organized as follows. First of all, we introduce the untwisted Kac-Moody algebras as central extensions of loop algebras. The algebra is naturally equipped with a Z Z-gradation, conventionally known as the homogeneous gradation. We then use a theorem of Kac to classify all the $\mathbf{Z}$-gradations of the algebra. Of special importance, from our point of view, are the subset of these gradations induced by elements of the Weyl group of the underlying finite Lie algebra. In these gradations there exist Heisenberg subalgebras whose elements have well defined grade. In fact, this leads to the crucial result that the inequivalent Heisenberg subalgebras, up to conjugation, are in one-to-one correspondence with conjugacy classes of the Weyl group. We point out that there is a natural notion of partial ordering on the space of $\mathbf{Z}$-gradations. A few trivial lemmas are also proved, which play an important rôle in later sections.

2.1. Untwisted Kac-Moody Algebras and Gradations. We will restrict our discussion to the untwisted Kac-Moody algebras, $\left(g^{(1)}\right.$ in the notation of $\mathrm{Kac}$ ), which can be realized as central extensions of the loop algebra of a finite Lie algebra $g$ :

$$
\hat{g}=\left(g \otimes \mathbf{C}\left[z, z^{-1}\right]\right) \oplus \mathbf{C} c \oplus \mathbf{C d} \text {. }
$$

If we write a typical element of $\hat{g}$ as $a_{n} \equiv\left(a \otimes z^{n}\right)$, where $a \in g$ and $n \in \mathbf{Z}$, then the algebra may be written as

$$
\begin{aligned}
{\left[a_{n}, b_{m}\right] } & =[a, b]_{n+m}+(a, b) c \delta_{n+m, 0}, \\
{\left[d, a_{n}\right] } & =n a_{n}, \\
{[d, d] } & =[c, d]=[c, c]=\left[c, a_{n}\right]=0,
\end{aligned}
$$

where $(a, b)$ is the Killing form of the finite Lie algebra $g$ and $[a, b]$ is the Lie-bracket in $g$. $c$ is the central element of $\hat{g}$, and $d$ is the derivation which naturally induces a $\mathbf{Z}$-gradation of $\hat{g}$ :

$$
\hat{g}=\bigoplus_{i \in \mathbf{Z}} \hat{g}_{i}
$$

where $\left[d, \hat{g}_{i}\right]=i \hat{g}_{i}$. This distinguished $\mathbf{Z}$-gradation is known as the homogeneous gradation.

At this time we point out the existence of a natural Heisenberg subalgebra of $\hat{g}$. Introducing the triangular decomposition of the finite Lie algebra $g=n_{-} \oplus h \oplus n_{+}$, allows us to define the homogeneous Heisenberg subalgebra as the algebra spanned by the elements $\left\{h \otimes z^{n}, c\right\}$ :

$$
\left[a_{n}, b_{m}\right]=(a, b) c \delta_{n+m, 0} \quad a, b \in h .
$$

This is a maximal commuting subalgebra of $\hat{g}$, with central extension. Clearly, a different choice of $h$ in $g$ will give an equivalent Heisenberg subalgebra, up to conjugation. One of the main purposes of Sect. 2.2 is to find all the inequivalent Heisenberg subalgebras of $\hat{g}$.

The classification of Z-gradations of $\hat{g}$ turns out to be equivalent to the problem of classifying all finite order inner automorphisms of $g$.

Theorem 2.1. (Kac [8,9]). Classification of all finite order inner automorphisms of g. Let $g$ be a (simple) Lie algebra of rank $r$, and $\mathbf{s}=\left(s_{0}, s_{1}, s_{2}, \ldots, s_{r}\right)$ be a sequence 
of non-negative relatively prime integers. Set

$$
N=\sum_{i=0}^{r} k_{i} s_{i}
$$

where $k_{i}$ are the Kac labels (that specify the decomposition of the highest root $\theta$ of $g$ in terms of the simple roots, that is $\theta=-\sum_{i=1}^{r} k_{i} \alpha_{i}$ and $k_{0}=1$ ). We then define the finite order inner automorphism $\sigma$ of $g$, in some Cartan-Weyl basis, by $\sigma: g \rightarrow g$ such that

$$
h \mapsto H, \quad E_{\alpha} \mapsto e^{2 \pi i \alpha \cdot \delta} E_{\alpha}
$$

where $\delta=\frac{1}{N} \sum_{i=1}^{r}\left(\frac{2}{\alpha_{i}^{2}}\right) s_{i} \omega_{i}$, and $\omega_{i}$ are the fundamental weights (these are the duals of the co-roots $\left.\alpha_{i}^{\vee}=2 \alpha_{i} / \alpha_{i}^{2}\right)$. Such automorphisms exhaust the finite order inner automorphisms of $g$ up to conjugacy.

An inner automorphism of $g$ can be used to define a new $\mathbf{Z}$-gradation of $\hat{g}$ in the following way. Under $\sigma, g$ has an eigenspace decomposition

$$
g=\bigoplus_{i} g_{i}(\sigma)
$$

where $\sigma: g_{k} \mapsto e^{2 \pi i k / N} g_{k}$ and $N$ is the order of $\sigma$. Consider the centrally extended loop algebra

$$
\hat{g}_{\sigma}=\bigoplus_{n \in \mathbf{Z}}\left(\bigoplus_{i} g_{i}(\sigma) \otimes z^{i+n N}\right) \oplus \mathbf{C} c \oplus \mathbf{C} d^{\prime},
$$

where the element $a \otimes z^{i+n N}$, for $a \in g_{i}(\sigma)$, of $\hat{g}_{\sigma}$ has grade $i+n N$ with respect to the derivation $d^{\prime}$.

Theorem 2.2. The algebra $\hat{g}_{\sigma}$ is isomorphic to $\hat{g}$ with a new derivation $d_{\sigma}$.

Proof. Theorem 2.1 implies that there exists a Cartan-Weyl basis for $g$ such that the inner automorphism acts as in (2.1). In this case

$$
\begin{aligned}
g_{0}(\sigma) & =h \cup\left\{E_{\alpha} \mid \alpha \cdot \delta=0\right\}, \\
g_{i}(\sigma) & =\left\{E_{\alpha} \mid \alpha \cdot \delta=i / N\right\} \quad i \neq 0,
\end{aligned}
$$

In order to exhibit the isomorphism we have to find a new derivation $d_{\sigma}$, of $\hat{g}$ such that

$$
\left[d_{\sigma}, a_{n}\right]=(i+n N) a_{n} \quad \forall a \in g_{i}(\sigma) .
$$

The required expression is $d_{\sigma}=N(d+\delta \cdot H)$, where $\delta$ is the vector of Theorem 2.1, and the isomorphism is, in this basis, $\left(a \otimes z^{n}\right) \in \hat{g} \cong\left(a \otimes z^{i+n N}\right) \in \hat{g}_{\sigma}$ for $a \in g_{i}(\sigma)$.

So we are led, following Kac $[8,9]$, to define the gradation of type $\mathbf{s}$.

Definition 2.1. A $\mathbf{Z}$-gradation of type $\mathbf{s}$ of $\hat{g}$ is defined in terms of a sequence of $r+1$ non-negative relatively prime integers $\mathbf{s}=\left(s_{0}, s_{1}, \ldots, s_{r}\right)$, by a derivation

$$
d_{\mathbf{s}}=\left(N d+\sum_{i=1}^{r}\left(\frac{2}{\alpha_{i}^{2}}\right) s_{i} \omega_{i} \cdot H\right) \text {. }
$$


Under $d_{\mathbf{s}}$ :

$$
\hat{g}=\bigoplus_{i \in \mathbf{Z}} \hat{g}_{i}(\mathbf{s})
$$

where $\left[d_{\mathbf{s}}, \hat{g}_{i}(\mathbf{s})\right]=i \hat{g}_{i}(\mathbf{s})$.

The homogeneous gradation is given by $\mathbf{s}=(1,0,0, \ldots, 0)$, in which case $\delta=0$. Another gradation of note is the principle gradation for which $s_{i}=1 \forall i$.

The $s_{i}$ 's are canonically associated to the nodes of the Dynkin diagram of $\hat{g}$ (the extended Dynkin diagram of $g$ ). There is a simple algorithm for determining the horizontal subalgebra, $\hat{g}_{0}(\mathbf{s})$, in a gradation of type $\mathbf{s}$. It is simply the algebra whose Dynkin diagram is found by removing nodes from the Dynkin diagram of $\hat{g}$ corresponding to non-zero $s_{i}$, and adding enough abelian factors to preserve the rank. So for the homogeneous gradation the horizontal algebra is $g$, whilst for the principle gradation it is the abelian algebra $h$.

Remark. Two gradations $\mathbf{s}$ and $\mathbf{s}^{\prime}$ are equivalent, up to trivial isomorphism, if there exists some symmetry of the Dynkin diagram of $\hat{g}$ that takes $\mathbf{s} \rightarrow \mathbf{s}^{\prime}$.

We will use the terminology "minimal gradation" to describe a gradation for which, for some $k, s_{k}=1$ and $s_{j}=0 \forall j \neq k$. Clearly the number of "minimal gradations" is equal to $r+1$, the number of nodes of the Dynkin diagram of $\hat{g}$; however, in view of the above remark not all such gradations are inequivalent if there exists some symmetry of the diagram. For example, the $A_{n}^{(1)}$ Dynkin diagram has a cyclic symmetry under which all nodes are equivalent, and so there is only one inequivalent "minimal gradation" of $A_{n}^{(1)}$.

2.2. Heisenberg Subalgebras and the Weyl Group. In this subsection we shall show how to classify all inequivalent Heisenberg subalgebras of $\hat{g}$. A Heisenberg subalgebra of $\hat{g}$ is a maximal nilpotent subalgebra (with central extension). The precise definition may be found in [10].

We have already established a connexion between inner automorphisms of $g$ and $\mathbf{Z}$-gradations of $\hat{g}$. There exists a certain gradation which is naturally associated to each Heisenberg subalgebra of $\hat{g}$, in the sense that elements of that Heisenberg subalgebra belong to the eigenspace of the derivation defining the gradation.

To this end, we are led to consider inner automorphisms of $g$ which fix a Cartan subalgebra $h^{\prime}$. Such automorphisms have the following action on the algebra $\hat{\rho}: g \rightarrow g$ (with $\hat{\rho}: h^{\prime} \rightarrow h^{\prime}$ ), so that in some Cartan-Weyl basis

$$
H^{\prime} \mapsto \rho\left(H^{\prime}\right), \quad E_{\alpha}^{\prime} \mapsto \psi_{\alpha} E_{\rho(\alpha)}^{\prime} .
$$

To avoid confusion later, we have used a prime to denote this Cartan-Weyl basis in order to distinguish it from the basis in (2.1). In the above $\rho$ is an automorphism of the root system $\Phi_{g}$ of $g$, and $\hat{\rho}=(\rho, \psi)$ is its lift into the algebra $g$. The automorphism group of $\Phi_{g}$, Aut $\left(\Phi_{g}\right)$ is a semi-direct product of the Weyl group of $g, W_{g}$, and the group of symmetries of the Dynkin diagram. We now restrict our attention to the Weyl group, the normal subgroup of $\operatorname{Aut}\left(\Phi_{g}\right)$; the diagram symmetries led to outer automorphisms of $g$ and we shall not discuss them further. So for every element $w \in W_{g}$ we have an inner automorphism of $g$ given by $\hat{w}: g \rightarrow g$ such that

$$
H^{\prime} \mapsto w\left(H^{\prime}\right), \quad E_{\alpha}^{\prime} \mapsto \psi_{\alpha} E_{w(\alpha)}^{\prime} .
$$


Notice that an element of the Weyl group acts naturally on the Cartan subalgebra. The phases $\psi_{\alpha}$ generate a projective representation of the root system with a factor set determined by the structure constants of $g$ :

$$
\psi_{\alpha} \psi_{\beta}=\frac{N_{\alpha, \beta}}{N_{w(\alpha), w(\beta)}} \psi_{\alpha+\beta}, \quad \psi_{\alpha} \psi_{-\alpha}=1 .
$$

The lift $\hat{w}$ is actually unique up to trivial automorphisms. The important point to notice about these inner automorphisms is that they fix the Cartan subalgebra of $g$, and it is because of this that they lead to classification of Heisenberg subalgebras of $\hat{g}$.

Theorem 2.3 (Kac and Peterson [10]). The lift $\hat{w}$ has order $N$ equal to $n$ or $2 n$, where $n$ is the order of $w$. When $w$ has no unit eigenvalues then $N=n$.

Remark. For the simply-laced algebras there is a rather simple way to determine whether the order of $\hat{w}$ is twice that of $w$, or not. It was shown in [12] that it is possible to find a basis for the phases such that the element $(\hat{w})^{n}$ acting on a step generator is

$$
(\hat{w})^{n} E_{\alpha} \mapsto(-)^{\alpha \cdot \tilde{\alpha}} E_{\alpha}
$$

where $\tilde{\alpha}=\sum_{p=1}^{n} w^{p}(\alpha)$. It follows that $\hat{w}$ has order $n$ or $2 n$ depending on whether $\alpha \cdot \tilde{\alpha} \in 2 \mathbf{Z} \forall \alpha \in \Phi_{g}$, or not. (Notice that if $w$ has no unit eigenvalues, then $\tilde{\alpha}=0 \forall \alpha \in \Phi_{g}$ and so $N=n$ as claimed.)

Under $\hat{w}, g$ has the following eigenspace decomposition

$$
g=\bigoplus_{i} g_{i}
$$

where $\hat{w}\left(g_{k}\right)=e^{2 \pi i k / N}\left(g_{k}\right)$. In particular, the Cartan subalgebra $h^{\prime}$ has an eigenspace decomposition $\bigoplus_{i \in I[w]} h_{i}^{\prime}$, where $I[w]$ is a set of $r=\operatorname{rank}(g)$ integers, such that the eigenvalues of $w$ are $\exp (2 \pi i k / N)$, for $k \in I[\omega]$. This set of numbers will be important in the following section and so we repeat its definition.

\section{Definition 2.2.}

$$
I[w]=\left\{k \in \mathbf{Z} \mid 0 \leqq k<N, \text { Eigenvalues of } w \text { are } e^{2 \pi i k / N}\right\} .
$$

Since $\hat{w}$ is an inner automorphism of $g$ there exists some different CartanWeyl basis (the unprimed basis) in which it acts as in (2.1). So for each element of the Weyl group there exists a unique, up to conjugation, inner automorphism of the form (2.1), and hence a Z-gradation of $\hat{g}$. We will denote the gradation corresponding to $w \in W_{g}$ as $\mathbf{s}[w]$. The gradation is unique (up to diagram symmetries) for conjugate elements of the Weyl group. Finding the gradation $\mathbf{s}[w]$ for a given conjugacy class of the Weyl group is not a straightforward task. Algorithms were suggested in [12], where they were used to find the gradations corresponding the 112 conjugacy classes of the Weyl group of $E_{8}$. (These results have been extended to other algebras in [13].)

The conjugacy classes of the Weyl group of all simple Lie algebras are given in a uniform way by Carter [11]. For each conjugacy class he gives a corresponding 
diagram, rather like a Dynkin diagram. Each node of the diagram corresponds to a root, not necessarily simple, whilst the number of lines joining two nodes equals $4 \cos ^{2} \Theta$, where $\Theta$ is the angle between the two roots. This gives two sorts of diagrams, some of which look exactly like Dynkin diagrams as well as a few exceptional diagrams that require their own notation. The element in the Weyl group represented by a given diagram is found by dividing the nodes into two sets $J_{1}$ and $J_{2}$ each containing mutually orthogonal roots and then performing Weyl reflections in all the roots in one set followed by Weyl reflections in each of the roots in the other. So if $R_{\beta}$ represents the Weyl reflection in the root $\beta$ then

$$
w=\left(\prod_{\beta \in J_{1}} R_{\beta}\right)\left(\prod_{\gamma \in J_{2}} R_{\gamma}\right),
$$

where the actual ordering in each set is irrelevant because in each set the roots are orthogonal. In particular, for the Coxeter element $w_{c}$ of $W_{g}$, the Carter diagram is the Dynkin diagram of $g$. For the $A_{n}$ algebras the Weyl group is isomorphic to the symmetric group on $n+1$ objects, $S_{n+1}$, and representatives of the conjugacy classes are found from the Carter diagrams which are simply the Dynkin diagrams for the subalgebras

$$
A_{n_{1}} \oplus A_{n_{2}} \oplus \cdots \oplus A_{n_{p}} \quad \text { such that } \quad \sum_{i=1}^{p}\left(n_{i}+1\right)=n+1
$$

where $A_{0}$ is taken to mean the empty set.

The important property of the gradations $\mathbf{s}[w]$, induced from conjugacy classes of the Weyl group, is that each of them has an associated Heisenberg subalgebra which is inequivalent, up to conjugacy, and moreover, these Heisenberg subalgebras completely exhaust the set of inequivalent Heisenberg subalgebras of $\hat{g}$.

Definition 2.3. The Heisenberg subalgebra $\mathscr{H}[w]$ of $\hat{g}$, corresponding to the representative $w$ of a conjugacy class of the Weyl group, is, in the basis for which $\hat{w}$ acts as in (2.2), simply the elements of $\hat{g}$ whose restriction to $g$ lies in the Cartan subalgebra $h^{\prime}$.

The important point is that elements of $\mathscr{H}[w]$ are homogeneous with respect to the gradation $d_{\mathbf{s}[w]}$. That is, $\mathscr{H}[w]$ has an eigenspace decomposition in $\mathbf{s}[w]$ gradation:

$$
\mathscr{H}[w]=\bigoplus_{i \in \mathbf{Z}} \mathscr{H}_{i}[w]
$$

where $\mathscr{H}_{j}[w]=\varnothing$ if $j \notin I[w] \bmod N$.

Remark. In the case when $c=0, \mathscr{H}[w]$ is a maximal commutative subalgebra of $\hat{g}$.

2.3. Partial Ordering of Gradations. In the construction which follows, it is important to be able to define a notion of partial ordering on the set of gradations. At this point we introduce some convenient notation.

$$
\hat{g}_{>i}(\mathbf{s})=\bigoplus_{k>i} \hat{g}_{k}(\mathbf{s})
$$

and similarly for $\hat{g}_{<i}(\mathbf{s}), \hat{g}_{\leqq i}(\mathbf{s})$ and $\hat{g}_{\geqq i}(\mathbf{s})$. 
Proposition 2.1. There exists a partial ordering on the set of gradations $\{\mathbf{s}\}$ of $\hat{g}$ such that $\mathbf{s} \geqq \mathbf{s}^{\prime}$ if $s_{i} \neq 0$ whenever $s_{i}^{\prime} \neq 0$.

For what follows we shall need the following lemmas relating to two gradations such that $\mathbf{s}^{\prime} \geqq \mathbf{s}$.

Lemma 2.1. For $\mathbf{s}^{\prime} \geqq \mathrm{s}$ :

$$
\hat{g}_{0}\left(\mathbf{s}^{\prime}\right) \subseteq \hat{g}_{0}(\mathbf{s})
$$

Lemma 2.2. For $\mathbf{s}^{\prime} \geqq \mathrm{s}:$

$$
\hat{g}_{j}(\mathbf{s}) \subset \begin{cases}\hat{g}_{>0}\left(\mathbf{s}^{\prime}\right) & j>0 \\ \hat{g}_{<0}\left(\mathbf{s}^{\prime}\right) & j<0 .\end{cases}
$$

Lemma 2.3. For $\mathbf{s}^{\prime} \geqq \mathbf{s}$ :

$$
\hat{g}_{j}\left(\mathbf{s}^{\prime}\right) \subset \begin{cases}\hat{g}_{\geqq 0}(\mathbf{s}) & j>0 \\ \hat{g}_{\leqq 0}(\mathbf{s}) & j<0 .\end{cases}
$$

The proof of the lemmas is trivial. Lemma 2.1 follows because when $\mathbf{s}^{\prime} \geqq \mathbf{s}$ the Dynkin diagram of $\hat{g}_{0}\left(\mathbf{s}^{\prime}\right)$ is a sub-diagram of the Dynkin diagram of $\hat{g}_{0}(\mathbf{s})$, by the removal of nodes. Lemma 2.2 follows because if $\alpha \cdot \delta_{\mathbf{s}}>0$, or $<0$, respectively, where $\delta_{\mathbf{s}}$ is the vector of Theorem 2.1 associated to $\mathbf{s}$, then this implies $\alpha \cdot \delta_{\mathbf{s}^{\prime}}>0$, or $<0$, respectively. While Lemma 2.3 follows because if $\alpha \cdot \delta_{\mathbf{s}^{\prime}}>0$, or $<0$, respectively, then this only implies $\alpha \cdot \delta_{\mathrm{s}} \geqq 0$, or $\leqq 0$, respectively.

\section{Generalized Hierarchies}

In this section we define a series of hierarchies of equations based on an untwisted Kac-Moody algebra with zero centre. The rather abstract construction will be made more concrete in the following section, where the usual Drinfel's-Sokolov hierarchies will be recovered as special cases. The method of defining the hierarchies and exhibiting the flows is just a generalization of the Drinfel'd-Sokolov matrix Lax representation approach [2], which itself extended earlier work [14]. As stated before, our approach is closely related to that of Wilson [3], in fact that reference contains the germ of idea that allows us to generalize the Drinfel'd-Sokolov hierarchies: that is, to consider more general automorphisms of the underlying Lie algebra. It will be clear that most of our arguments are direct generalizations of those of [2]. Throughout the rest of the paper we are considering $\hat{g}$ to have zero centre $c=0$.

3.1. Type I Hierarchies. We begin by defining a differential operator $L$, associated to the data $(\Lambda, w, \mathbf{s})$, in the space $C^{\infty}(\mathbf{R}, \hat{g})$, where $\hat{g}$ is an untwisted Kac-Moody algebra:

$$
L=\partial_{x}+q+\Lambda
$$

In the above we require:

(1). $\Lambda$ to be a constant regular element of $\mathscr{H}_{i}[w]$ with $i>0$ in the gradation $\mathbf{s}[w]$ of $\hat{g}$. $w$ is some element of $W_{g}$, and conjugate elements of $W_{g}$ will lead to isomorphic 
hierarchies. By constant we mean $\partial_{x} \Lambda=0$, and by regular we mean that $\operatorname{Ker}(\operatorname{ad} \Lambda)=$ $\mathscr{H}[w]$ (i.e. in its restriction to $g, \operatorname{Ker}\left(\left.\operatorname{ad} \Lambda\right|_{g}\right)$ is a Cartan subalgebra). This will not be the case if $\Lambda$ lies in a wall, that is a hyperplane perpendicular to some root. In such a case $\left[\Lambda, E_{\alpha}\right]=0$, for the particular root $\alpha$, and so the $\operatorname{Ker}\left(\left.\operatorname{ad} \Lambda\right|_{g}\right)$ would be larger than $h$. We shall refer to the hierarchies which satisfy this constraint as type-I hierarchies. For type-II hierarchies $\Lambda$ is no longer regular, however, one can still construct an infinite hierarchy of integrable equations. We shall, for the moment, concentrate on type-I hierarchies, we shall discuss type-II hierarchies briefly in Sect. 3.3.

(2). The potential $q \in C^{\infty}(\mathbf{R}, Q)$, where $Q$ is defined to be the following subspace of $\hat{g}$ :

$$
Q=\hat{g}_{\geqq 0}(\mathbf{s}) \cap \hat{g}_{<i}(\mathbf{s}[w]),
$$

using the notation of (2.3). It is convenient to define

$$
Q^{(j)}=\hat{g}_{\geqq 0}(\mathbf{s}) \cap \hat{g}_{<j}(\mathbf{s}[w]),
$$

and so $Q \equiv Q^{(i)}$. In the above, $\mathbf{s}$ is some other gradation of $\hat{g}$ such that $\mathbf{s} \leqq \mathbf{s}[w]$.

Proposition 3.1. There exist an infinite number of commuting flows of the form

$$
\frac{\partial L}{\partial t_{b}}=[A(b), L],
$$

where $b$ is a constant element of $\mathscr{H}_{+}[w]$, the subspace of $\mathscr{H}[w]$ with positive $\mathbf{s}[w]$-grade, and $A(b) \in C^{\infty}(\mathbf{R}, \hat{g})$ will be defined below.

The fact that the flows commute means that it makes sense to define $q$ as a function of all the flows: $q\left(x, \ldots, t_{b}, \ldots\right)$. (Following the philosophy of [2] we shall treat the $t_{b}$ 's in the calculations below as parameters and temporally "forget" that the potentials $A(b)$ and $q$ depend on them.)

The elucidation of Proposition 3.1 will require a couple of lemmas which we now establish.

Definition 3.1. Given some $M=\sum_{j=-\infty}^{p} m_{j}=\sum_{j=-\infty}^{p^{\prime}} m_{j}^{\prime}$, where $m_{j} \in C^{\infty}\left(\mathbf{R}, \hat{g}_{j}(\mathbf{s})\right)$ and $m_{j}^{\prime} \in C^{\infty}\left(\mathbf{R}, \hat{g}_{j}(\mathbf{s}[w])\right)$ are the expansion coefficients in the two gradations, we shall define $M_{+}=\sum_{j=0}^{p} m_{j}, M_{-}=M-M_{+}, M_{+}^{\prime}=\sum_{j=0}^{p^{\prime}} m_{j}^{\prime}$ and $M_{-}^{\prime}=M-M_{+}^{\prime}$.

Lemma 3.1. If $[M, L]=0$, where $M$ has the form of Definition 3.1, then

$$
\left[M_{+}, L\right] \in C^{\infty}(\mathbf{R}, Q),
$$

and

$$
\left[M_{+}^{\prime}, L\right] \in C^{\infty}(\mathbf{R}, Q) .
$$

Proof. The proof proceeds by equating the grades on the left- and right-hand sides of the equalities $\left[M_{+}, L\right]=-\left[M_{-}, L\right]$ and $\left[M_{+}^{\prime}, L\right]=-\left[M_{-}^{\prime}, L\right]$. At worst, the 
grades of the various terms are:

\begin{tabular}{|l|l|l|}
\hline & $\mathbf{s}$ & $\mathbf{s}[w]$ \\
\hline$L$ & 0 to $\infty$ & $-\infty$ to $i$ \\
$M_{+}$ & 0 to $\infty$ & $-\infty$ to $\infty$ \\
$M_{-}$ & $-\infty$ to -1 & $-\infty$ to -1 \\
$M^{\prime}$ & 0 to $\infty$ & 0 to $\infty$ \\
$M_{-}^{\prime^{+}}$ & $-\infty$ to 0 & $-\infty$ to -1 \\
\hline
\end{tabular}

where use was made of Lemmas $2.1,2.2$, and 2.3 , which apply because $\mathbf{s}[w] \geqq \mathbf{s}$. So, for example, $-\left[M_{-}, L\right]$ has s-grade in the range $(-\infty$ to $\infty)$ and $\mathbf{s}[w]$-grade in the range $(-\infty$ to $i-1)$, whilst $\left[M_{+}, L\right]$ has s-grade in the range $(0$ to $\infty)$ and $\mathbf{s}[w]$-grade in the range $(-\infty$ to $\infty)$. Since these two expressions must have the same grade, the actual grade of both expressions lies in the intersection, which proves (3.3). Similarly one proves (3.4).

Definition 3.2. There exists a formal action of the Kac-Moody group $\hat{G}$ on $L$, which we call a gauge transformation. The action is defined by the exponentiated adjoint action:

$$
L_{0}=\exp (\operatorname{ad} T)(L), \quad T \in C^{\infty}(\mathbf{R}, \hat{g}),
$$

where the exponentiated adjoint action is defined by its power series expansion, i.e.

$$
\exp (\operatorname{ad}(T))(a)=a+[T, a]+\frac{1}{2}[T,[T, a]]+\frac{1}{3 !}[T,[T,[T, a]]]+\cdots
$$

Proposition 3.2. There exists a (non-unique) gauge transformation of the form given above, where $T \in C^{\infty}\left(\mathbf{R}, g_{<0}(\mathbf{s}[w])\right)$, such that $L_{0}$ has the form

$$
L_{0}=\partial_{x}+\Lambda+\sum_{j<i} H_{j}
$$

where $H_{j} \in C^{\infty}\left(\mathbf{R}, \mathscr{H}_{j}[w]\right)$.

Proof. Essentially one equates terms of the same $\mathbf{s}[w]$-grade in the expression $L_{0}=$ $\exp (\operatorname{ad}(T))(L)$, to get a recursion relation of the form

$$
H_{j}+\left[\Lambda, T_{j-i}\right]=\star \text {, }
$$

where $T_{j}$ is the component of $T$ of $\mathbf{s}[w]$-grade $j$, and $\star$ denotes terms of grade $j$ which depend on $H_{k}$, for $k>j, T_{k}$, for $k>j-i$, and $q$. The proof proceeds by induction. The first equation of the series states that

$$
H_{i-1}+\left[\Lambda, T_{-1}\right]=q_{i-1},
$$

where $q_{i-1}$ is the component of $q$ of $\mathbf{s}[w]$-grade $i-1$. We now appeal to the fact that $\Lambda$ is a regular element. This implies that $\hat{g}$ has the decomposition $\hat{g}=\operatorname{Ker}(\operatorname{ad} \Lambda) \oplus$ $\operatorname{Im}(\operatorname{ad} \Lambda)$, where $\operatorname{Ker}(\operatorname{ad} \Lambda)=\mathscr{H}[w]$. Hence, we can solve (3.7) uniquely for $H_{i-1}$, and for $T_{-1}$, up to an element of $\operatorname{Ker}(\operatorname{ad} \Lambda)$. We can fix the freedom in the $T_{j}$ 's by demanding that $T_{j} \in \operatorname{Im}(\operatorname{ad} \Lambda)$. The same decomposition means that we can solve (3.6) iteratively. 
Proof of Proposition 3.1. We have to find $A$ 's such that the right-hand side of (3.2) is in $C^{\infty}(\mathbf{R}, Q)$. By Lemma 3.1 if we can find $M$ such that $[M, L]=0$, then suitable expressions for $A$ are $M_{+}$or $M_{+}^{\prime}$. Using the fact that $\exp (\operatorname{ad} T)[M, L]=$ $\left[\exp (\operatorname{ad} T) M, L_{0}\right]$, where $T$ was found in Proposition 3.2, we can find $M$ 's which commute with $L$ by using the fact that $L_{0}$ consists of a derivative and terms in $\mathscr{H}[w]$. So we get the two sets of flows

$$
\begin{aligned}
& \frac{\partial L}{\partial t}=\left[(\exp (-\operatorname{ad} T)(b))_{+}, L\right], \\
& \frac{\partial L}{\partial t^{\prime}}=\left[(\exp (-\operatorname{ad} T)(b))_{+}^{\prime}, L\right] .
\end{aligned}
$$

for a constant element $b \in \mathscr{H}_{+}[w]$.

Now we introduce the concept of a gauge symmetry of the system of equations. If $\mathbf{s} \prec \mathbf{s}[w]$ them the form of $L$ is preserved under certain gauge transformations. In this case one can define a reduced set of flows which preserve some consistent slice of the orbits of the symmetry.

Proposition 3.3. The form of $L$ is preserved under certain gauge transformations. In fact the most general such gauge transformation, $L^{\prime}=\exp (\operatorname{ad} S)(L)$, has $S \in C^{\infty}(\mathbf{R}, P)$, where

$$
P=\hat{g}_{0}(\mathbf{s}) \cap \hat{g}_{<0}(\mathbf{s}[w])
$$

Under this action:

$$
q \mapsto \exp (\operatorname{ad} S)\left(\partial_{x}+q+\Lambda\right)-\partial_{x}-\Lambda
$$

Proof. Consider an infinitesimal gauge transformation. Under such a transformation

$$
\delta q=-\partial_{x} S+[S, q+\Lambda] .
$$

Requiring that $\delta q \in C^{\infty}(\mathbf{R}, Q)$ implies that $S$ has to have $\mathbf{s}[w]$-grade $<0$ and s-grade $\geqq 0$, and so $S \in C^{\infty}(\mathbf{R}, P)$.

Furthermore, $\exp (\operatorname{ad} S)$ acts freely owing to the fact that the map ad $\Lambda: P \rightarrow Q$ is injective. (This follows because it is easily shown that $P$ contains no elements in $\operatorname{Ker}(\operatorname{ad} \Lambda)$.) It is therefore possible to fix the gauge freedom by choosing some consistent gauge slice of $C^{\infty}(\mathbf{R}, Q) / C^{\infty}(\mathbf{R}, P)$, say $C^{\infty}(\mathbf{R}, \widetilde{Q})$. The dimension of $\widetilde{Q}$ is easy to calculate

$$
\begin{aligned}
\operatorname{dim}(\tilde{Q}) & =\operatorname{dim}(Q)-\operatorname{dim}(P) \\
& =\operatorname{dim}\left(\hat{g}_{\geqq 0}(\mathbf{s}) \cap\left(\bigoplus_{j=0}^{i-1} \hat{g}_{j}(\mathbf{s}[w])\right)\right) \\
& =\operatorname{dim}\left(\bigoplus_{j=0}^{i-1} \hat{g}_{j}(\mathbf{s}[w])\right),
\end{aligned}
$$

by Lemma 2.3. Notice that the dimension of $\widetilde{Q}$ is independent of the gradation $\mathbf{s}$.

We can now write consistent flows for the gauge fixed operator $\widetilde{L}$, by choosing $q \in C^{\infty}(\mathbf{R}, \widetilde{Q})$. However, if we do this then there is no guarantee that (3.8) and (3.9) will preserve the gauge slice chosen. To compensate for this it will be necessary 
to modify (3.8) and (3.9) by infinitesimal gauge transformations:

$$
\begin{aligned}
& \frac{\partial \tilde{L}}{\partial t}=\left[(\exp (-\operatorname{ad} T)(b))_{+}+\theta, \tilde{L}\right], \\
& \left.\frac{\partial \tilde{L}}{\partial t^{\prime}}=[\exp (-\operatorname{ad} T)(b))_{+}^{\prime}+\theta^{\prime}, \tilde{L}\right],
\end{aligned}
$$

for some $\theta, \theta^{\prime} \in C^{\infty}(\mathbf{R}, P)$.

Proposition 3.4. (3.11) and (3.12) lead to identical equations for $\tilde{q}$.

Proof. Consider $M$ an element of $\hat{g} . M_{+}-M^{\prime}{ }_{+}$clearly has $\mathbf{s}[w]$-grade less than zero, by Lemmas 2.1 and 2.3, and moreover by Lemma 2.2 it has s-grade equal to zero. Hence, $M_{+}-M_{+}^{\prime} \in P$. Applying this result, we see that the difference $(\exp (-\operatorname{ad} T)(b))_{+}-(\exp (-\operatorname{ad} T)(b))_{+}^{\prime}$ is an element of $C^{\infty}(\mathbf{R}, P)$, but neither (3.11) nor (3.12) evolves $\tilde{L}$ out of the gauge slice $\tilde{Q}$, therefore neither can their difference. This implies that

$$
(\exp (-\operatorname{ad} T)(b))_{+}-(\exp (-\operatorname{ad} T)(b))_{+}^{\prime}+\theta-\theta^{\prime}=0,
$$

and therefore the difference of the right-hand sides of (3.11) and (3.12) is identically zero, and so $t \equiv t^{\prime}$.

Definition 3.3. If we introduce a basis $\xi_{a}, a=1, \ldots, \operatorname{dim}(\tilde{Q})$ for $C^{\infty}(\mathbf{R}, \tilde{Q})$, and a basis for $\mathscr{H}_{+}[w]$ consisting of $\Lambda_{j, n} \in \mathscr{H}_{j+n N}[w]$, with $j \in I[w]$ and $n \in \mathbf{Z} \geqq 0$, then we call the hierarchy of flows of (3.11) (or (3.12)) with $b=\Lambda_{j, n}$, which can be written in the form

$$
\frac{\partial \xi_{a}}{\partial t_{j, n}}=f_{a}^{(j, n)}\left(\xi_{b}, \partial_{x} \xi_{b}, \partial_{x}^{2} \xi_{b}, \ldots\right) \quad j \in I[w], \quad n \in \mathbf{Z} \geqq 0,
$$

the $(\Lambda, \mathbf{s}[w], \mathbf{s})$-hierarchy, with a particular choice of gauge, associated to $\hat{g}$. The hierarchies corresponding to conjugate elements of $W_{g}$ are isomorphic.

In the following couple of propositions we establish that the flows defined by (3.2) commute. We also find the conserved densities of the hierarchy, and show that some components of $q$ are constant under all the flows and may be consistently set to zero.

Proposition 3.5. The quantities $H_{j}, j \in I \bmod N \mathrm{Z}<i$, of (3.5) are the conserved densities for the hierarchy.

Proof. Since $\hat{g}$ is a loop algebra we shall consider a particular faithful matrix representation of $g$. Recall that there exists a gauge transformation $T$ such that $L_{0}=\exp (\operatorname{ad}(T))(L)$, has the form:

$$
L_{0}=\partial_{x}+\Lambda+\sum_{j<i} H_{j}
$$

where $H_{j} \in C^{\infty}\left(\mathbf{R}, \mathscr{H}_{j}[w]\right)$. Now that we have a definite matrix representation in mind, the gauge transformations may then be written as $L_{0}=\Phi L \Phi^{-1}$, where $\Phi$ is a matrix with an expansion of the form $\Phi=\mathbf{I}+\sum_{j<0} \Phi_{j}$, where $\Phi_{j}$ has $\mathbf{s}[w]$-grade $j$. The $\Phi_{j}$ 's are polynomials of the $T_{j}$ 's of Proposition 3.2. Each flow of the hierarchy 
can be written in the form

$$
\left[\partial_{t}-\tilde{A}, L_{0}\right]=0
$$

where for each flow

$$
\tilde{A}=\left(\partial_{t} \Phi\right) \Phi^{-1}+\Phi A \Phi^{-1} .
$$

It is easy to see that (3.13) requires the component $\tilde{A}_{j} \in C^{\infty}\left(\mathbf{R}, \mathscr{H}_{j}[w]\right)$, and so (3.13) has the form of a conservation equation

$$
\partial_{t} H_{j}+\partial_{x} \tilde{A}_{j}=0
$$

Proposition 3.6. The finite set of $H_{j}$ 's with $i>j \geqq 0$ are constant along all the flows (recall that $i$ is defined by the fact that $\Lambda \in \mathscr{H}_{i}[w]$ ), i.e. for any flow

$$
\partial_{t} H_{j}=0 \quad i>j \geqq 0 .
$$

Proof. Consider (3.14) and the fact that the explicit expression for $A$ is

$$
A=\left(\Phi^{-1} b \Phi\right)_{+}^{\prime}+\theta^{\prime}
$$

This can be rewritten as

$$
A=\Phi^{-1} b \Phi-\left(\Phi^{-1} b \Phi^{\prime}\right)_{-}+\theta^{\prime}
$$

and so

$$
\tilde{A}=\left(\partial_{t} \Phi\right) \Phi^{-1}+b-\Phi\left(\left(\Phi^{-1} b \Phi\right)_{-}^{\prime}-\theta^{\prime}\right) \Phi^{-1}
$$

The only term in the above with $\mathbf{s}[w]$-grade $\geqq 0$ is $b$, which is a constant element of $\mathscr{H}_{+}[w]$, and therefore $\partial_{x} \tilde{A}_{j}=0 \forall j \geqq 0$.

This result means that some components of $q$ can be eliminated, since they are constant under the flows. For example, (3.7) implies that

$$
H_{i-1}=q_{i-1} \cap C^{\infty}(\mathbf{R}, \mathscr{H}[w]),
$$

and so by Proposition 3.6 we can consistently set

$$
q_{i-1} \cap C^{\infty}(\mathbf{R}, \mathscr{H}[w])=0 .
$$

Indeed one can show directly that $\partial_{t} q_{i-1}=\left[\Lambda, T_{-1}\right]$.

Proposition 3.7. The flows defined by (3.2) commute for different elements $b$ and $b^{\prime} \in \mathscr{H}_{+}[w]$.

Proof. It will be sufficient for us to show that the flows on $L$ before gauge fixing commute. The condition that the flows commute:

$$
\left[\frac{\partial}{\partial t}, \frac{\partial}{\partial t^{\prime}}\right] L=0,
$$

will be satisfied if

$$
\partial_{t} A\left(b^{\prime}\right)_{+}-\partial_{t^{\prime}} A(b)_{+}+\left[A\left(b^{\prime}\right)_{+}, A(b)_{+}\right]=0,
$$

where $A(b)=\Phi^{-1} b \Phi$. In Proposition 3.5 we showed that $\tilde{A}$, defined in Eq. (3.14), is an element of $\mathscr{H}[w]$, and so $\left[\partial_{t}-\tilde{A}(b), b^{\prime}\right]=0$, since $b^{\prime}$ is a constant element of 
$\mathscr{H}[w]$, and similarly $\left[\partial_{t^{\prime}}-\tilde{A}\left(b^{\prime}\right), b\right]=0$. By conjugating these with $\Phi$ we deduce

$$
\partial_{t} A\left(b^{\prime}\right)=\left[A(b)_{+}, A\left(b^{\prime}\right)\right], \quad \partial_{t}^{\prime} A(b)=\left[A\left(b^{\prime}\right)_{+}, A(b)\right],
$$

and so $\partial_{t} A\left(b^{\prime}\right)_{+}=\left[A(b)_{+}, A\left(b^{\prime}\right)\right]_{+}$, and similarly for $\partial_{t^{\prime}} A(b)_{+}$. Substituting into the left-hand side of (3.17), we find

$$
\left[A\left(b^{\prime}\right)_{+}, A(b)\right]_{+}-\left[A(b)_{+}, A\left(b^{\prime}\right)\right]_{+}+\left[A(b)_{+}, A\left(b^{\prime}\right)_{+}\right] .
$$

But $\left[A\left(b^{\prime}\right)_{+}, A(b)\right]_{+}=\left[A(b), A\left(b^{\prime}\right)_{-}\right]_{+}=\left[A(b)_{+}, A\left(b^{\prime}\right)_{-}\right]_{+}$and so (3.16) is zero.

Proposition 3.8. The (gauge-fixed) flows (3.11) (or (3.12)) have the form

$$
\frac{\partial \tilde{L}}{\partial t_{j, n}}=\left[A_{j, n}, \tilde{L}\right],
$$

where $A_{j, n}=\Lambda_{j, n}+q_{j, n}, \Lambda_{j, n}$ is the same as in definition 3.3 and $q_{j, n} \in C^{\infty}\left(\mathbf{R}, Q^{(n N+j)}\right)$, where $Q^{(k)}$ was defined in (3.1).

Proof. $A_{j, n}$ has the form

$$
\begin{aligned}
A_{j, n} & =\left(\exp -\operatorname{ad} T\left(\Lambda_{j, n}\right)\right)_{+}+\theta \\
& =\left(\exp -\operatorname{ad} T\left(\Lambda_{j, n}\right)\right)_{+}^{\prime}+\theta^{\prime} .
\end{aligned}
$$

By considering the grades of the two expressions, one deduces that $A_{j, n}$ has $\mathbf{s}[w]$-grade $\leqq j+n N$ and s-grade $\geqq 0$. But the component of $A_{j, n}$ with $\mathbf{s}[w]$-grade equal to $j+n N$ is $\Lambda_{j, n}$ and therefore the rest of $A_{j, n}$, that is $q_{j, n}$, has grades lying in the intersection $\hat{g}_{\geqq 0}(\mathbf{s}) \cap \hat{g}_{<j+n N}(\mathbf{s}[w])$.

As a consequence of Proposition 3.8 we see that the hierarchy has a rather uniform description. If we define

$$
L_{j, n}=\partial_{t_{j, n}}-q_{j, n}-\Lambda_{j, n} \quad j \in I[w], \quad n \in \mathbf{Z} \geqq 0,
$$

where the original $L$-operator is $L=-L_{k, m}$, with $x=-t_{k, m}, q=q_{k, m}$ and $\Lambda=\Lambda_{k, m}$, for some fixed $k$ and $m$, such that $k+m N=i$, the grade of $\Lambda$. In the above, $\Lambda_{j, n} \in \mathscr{H}_{j+n N}[w]$ and constant, and the potential $q_{j, n} \in C^{\infty}\left(\mathbf{R}, Q^{(j+n N)}\right)$. The equations of the hierarchy, after suitable gauge fixing, lead to the zero-curvature conditions

$$
\left[L_{k, m}, L_{j, n}\right]=0 \text {. }
$$

3.2. Generalized Miura Maps. We now consider the spectrum of hierarchies for fixed $w$ and $\Lambda \in \mathscr{H}_{+}[w]$. The spectrum results from varying the gradation $\mathbf{s} \leqq \mathbf{s}[w]$. For $\mathbf{s}=\mathbf{s}[w]$, where by "equals" we mean as vectors, then it is apparent from (3.10) that there is no gauge invariance and so we have a generalization of the Drinfel'dSokolov modified-KdV hierarchies. On the contrary, when s is as "small" as it can be, i.e. a "minimal gradation," the gauge invariance is maximal, then we have a generalization of the Drinfel'd-Sokolov KdV hierarchies. Choosing a gradation $\mathbf{s}$ between these two extremes leads to what we will call a partially modified $\mathrm{KdV}$ hierarchy $(\mathrm{pmKdV})$. We will show that there exist generalized Miura maps connecting some of these hierarchies in the spectrum.

Proposition 3.9. For the hierarchies of fixed $w$ and $\Lambda$, the two hierarchies defined by $L_{1}$ and $L_{2}$ corresponding to gradations $\mathbf{s}_{1}$ and $\mathbf{s}_{2}$, respectively, with $\mathbf{s}[w] \geqq \mathbf{s}_{1} \succ \mathbf{s}_{2}$, 
are related by the fact that $L_{1}$ can be obtained by appropriately restricting the potential of $L_{2}$.

Proof. First of all $Q_{1} \subset Q_{2}$. This follows from Lemmas 2.1 and 2.3, which apply because $\mathbf{s}_{1}>\mathbf{s}_{2}$. Let us denote the complement $X: Q_{2}=Q_{1} \oplus X$. It follows from Lemmas 2.1 and 2.3 that

$$
X=\hat{g}_{0}\left(\mathbf{s}_{2}\right) \cap \hat{g}_{<0}\left(\mathbf{s}_{1}\right) .
$$

We now restrict $L_{2}$ by imposing $q_{2} \cap C^{\infty}(\mathbf{R}, X)=0$, and so $L_{2} \rightarrow L_{1}$.

Proposition 3.9 allows us to introduce the concept of a generalized Miura transform. If we have a solution of the $\left(\Lambda, w, \mathbf{s}_{1}\right)$-hierarchy, then we can translate this into a solution of any $\left(\Lambda, w, \mathbf{s}_{2}\right)$-hierarchy by a suitable gauge transformation, where $\mathbf{s}_{1}>\mathbf{s}_{2}$.

3.3. Type II Hierarchies. Type II hierarchies are defined by the fact that $\Lambda$, the constant element of $\mathscr{H}_{+}[w]$, is not regular. As has already been pointed out, this will occur if, in the basis of (2.2), where $\Lambda=\mu \cdot H^{\prime}$ for some $\mu, \mu$ lies in some walls $\left\{W_{\alpha}\right\}$ (these are the hyperplanes orthogonal to a given $\alpha \in \Phi_{g}$ ). If this does occur then $\operatorname{Ker}(\operatorname{ad} \Lambda)$ is now larger than $\mathscr{H}[w]$.

It is rather obvious how to modify the construction of the type I hierarchies to that of type II hierarchies. Following the same steps, the conserved densities $H_{j}$ in (3.5) are now elements of $\operatorname{Ker}(\operatorname{ad} \Lambda)$, and a set of commuting flows can be constructed of the form (3.2), but now $b$ is constrained to lie in the centre of $\operatorname{Ker}(\operatorname{ad} \Lambda)$, a point discussed in ref. [3].

\section{Examples}

In this section, a number of examples of the preceding formalism will be developed. Our examples will be restricted to type I hierarchies.

4.1. The Drienfel'd-Sokolov Hierarchies. The hierarchies defined in ref. [2] can be recovered from a $(\Lambda, w, \mathbf{s})$-hierarchy by choosing $w$ to be Coxeter element $w_{c}$ of $W_{g}$, and $\Lambda$ to be the (unique) element of $\hat{g}_{1}\left(\mathbf{s}\left[w_{c}\right]\right)$. The Coxeter element of $W_{g}$ was discussed in Sect. 2.2. Recall that $I\left[w_{c}\right]=\{$ Exponents of $g\}$ and the order of $w_{c}$ is the Coxeter number $h$ of $g$. For this element of $W_{g}$ the lift $\hat{w}_{c}$ also has order $h$, because $w_{c}$ has no unit eigenvalues. The gradation associated to $w_{c}$ is the principle gradation for which $\mathbf{s}\left[w_{c}\right]=(1,1, \ldots, 1)$. For this choice of gradation the whole of $\mathscr{H}\left[w_{c}\right]$ is regular, and so the hierarchies are of type $\mathrm{I}$, in this case $\hat{g}=\operatorname{Ker}(\operatorname{ad} \Lambda) \oplus \operatorname{Im}(\operatorname{ad} \Lambda)$. In order to make contact with [2] we shall write down the expression for the $L$-operator in the basis in which the Coxeter automorphism is the adjoint action of an element of the Cartan subalgebra. This is the basis of (2.1). $\hat{g}_{1}$ is spanned by the $r+1$ elements $e_{i}, i=0, \ldots, r$, where

$$
e_{i}=E_{\alpha_{i}}, \quad e_{0}=z E_{-\alpha_{0}},
$$

where the $\alpha_{i}$ are the sample roots of $g$ and $\alpha_{0}$ is the extended root. The spectrum of the Heisenberg algebra is

$$
\mathscr{H}\left[w_{c}\right]=\bigoplus_{j \in I\left[w_{c}\right] \bmod h \mathbf{Z}} \mathscr{H}_{j}\left[w_{c}\right] .
$$


The unique element of the Heisenberg algebra in $\hat{g}_{1}\left[w_{c}\right]$ is

$$
\Lambda=\sum_{i=0}^{r} e_{i}
$$

For fixed, $g$ we have a spectrum of hierarchies by varying the gradation $\mathbf{s} \leqq \mathbf{s}\left[w_{c}\right]$. The usual generalized $\mathrm{mKdV}$ hierarchy is recovered choosing $\mathbf{s}=\mathbf{s}\left[w_{c}\right]$, in which case

$$
L=\partial_{x}+q+\Lambda
$$

where $\Lambda$ is $(4.1)$ and $q \in C^{\infty}\left(\mathbf{R}, g_{0}\left(\mathbf{s}\left[w_{c}\right]\right) \equiv h\right)$. The usual generalized $\mathrm{KdV}$ hierarchy is recovered by choosing $\mathbf{s}$ to be a "minimal gradation," i.e. $s_{k}=1$ and $s_{j}=0 \forall j \neq k$, for some $k$. So there is a $\mathrm{KdV}$ hierarchy for each node of the extended Dynkin diagram, up to symmetries of the diagram. In this case $q \in C^{\infty}(\mathbf{R}, Q)$ where

$$
Q=\hat{g}_{0}(\mathbf{s}) \cap \hat{g}_{\leqq 0}\left(\mathbf{s}\left[w_{c}\right]\right) .
$$

The gauge freedom corresponds to $C^{\infty}(\mathbf{R}, P)$, where

$$
P=\hat{g}_{0}(\mathbf{s}) \cap \hat{g}_{<0}\left(\mathbf{s}\left[w_{c}\right]\right) .
$$

Using the Drinfel'd-Sokolov formalism one can construct the flows $t_{j, n}$, labelled by $j \in I\left[w_{c}\right] \equiv\{$ Exponents $\}$ and $n \in \mathbf{Z} \geqq 0$. Reference [2] discusses a rather natural way to gauge fix the KdV hierarchies. For an arbitrary, "non-minimal gradation" $\mathbf{s} \prec \mathbf{s}\left[w_{c}\right]$, the hierarchies are partially modified.

For the $g=A_{n}$ hierarchies, which include the usual $\mathrm{mKdV}$ and $\mathrm{KdV}$ hierarchies when $n=1$, we can write down the $L$-operator explicitly in the $n+1$-dimensional representation. In this representation, $E_{\alpha_{1}}=e_{i+1 i}$, (the matrix with a 1 in the $i^{\text {th }}$ row and $i+1^{\text {th }}$ column, and zeros elsewhere), and so

$$
\Lambda=\left(\begin{array}{ccccc}
0 & 1 & 0 & \cdots & 0 \\
\vdots & \ddots & 1 & \ddots & \vdots \\
\vdots & & \ddots & \ddots & 0 \\
0 & & & \ddots & 1 \\
z & 0 & \cdots & \cdots & 0
\end{array}\right)
$$

The Heisenberg subalgebra is spanned by elements which can be written in block form as

$$
\Lambda_{j, m}=z^{m}\left(\begin{array}{cc}
\mathbf{0} & \mathbf{I}_{n+1-j} \\
z \mathbf{I}_{j} & \mathbf{0}
\end{array}\right) \in \mathscr{H}_{j+m n}\left[w_{c}\right], \quad j=1,2, \ldots, n, \quad m \in \mathbf{Z},
$$

where $\mathbf{I}_{i}$ is the $i \times i$ unit matrix. The $\mathrm{mKdV} L$-operator is

$$
L=\partial_{x}+\left(\begin{array}{cccc}
v_{1} & & & \\
& v_{2} & & \\
& & \ddots & \\
& & & v_{n+1}
\end{array}\right)+\left(\begin{array}{ccc} 
& 1 & \\
& \ddots & \\
& & 1 \\
z & &
\end{array}\right)
$$

where $\sum_{j=1}^{n+1} v_{j}=0$. The usual choice of gauge fixing leads to an $L$-operator for the 
generalized $A_{n}-\mathrm{KdV}$ of the form

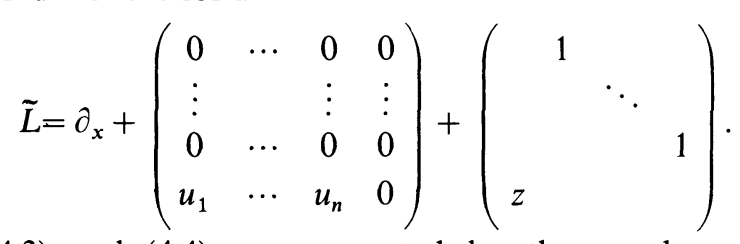

Solutions of (4.3) and (4.4) are connected by the usual generalized Miura $\operatorname{map}\left\{v_{i}\right\} \mapsto\left\{u_{i}\right\}$.

4.2. Fractional $\mathrm{KdV}$-Hierarchies. Recently, a new series of integrable hierarchies has been proposed [15], called the fractional KdV hierarchies (FKdV). Their structure evolved from a generalization of $W$-algebra proposed in [7]. (These algebras are related to the Hamiltonian structure of the hierarchies.) The idea is to generalize the Drinfel'd-Sokolov construction, for the case $g=A_{n}$, to $L$-operators of the form

$$
L=\partial_{x}+q+\Lambda_{i, 0},
$$

where $\Lambda_{i, 0}$ is given in (4.2). From our point of view this is nothing but the $\left(\Lambda, w_{c}, \mathbf{s}\right)$ hierarchy, where in contrast to the usual case $\Lambda$ is chosen to be the unique element of the Heisenberg algebra in $\hat{g}_{i}\left[w_{c}\right]$, where $1 \leqq i \leqq n$. In fact, one could consider more general situations in which $\Lambda$ was any of the elements in (4.2) with $m \geqq 0$. Such a generalization is possible even for the standard $S L(2)-\mathrm{KdV}$ hierarchy, a direction which will be pursued elsewhere.

For the case of $A_{2}$ the proposed form of $L$ is $[7,15]$

$$
L=\partial_{x}+\left(\begin{array}{ccc}
(\alpha-\beta) U & 0 & 0 \\
G^{+} & -\alpha U & 0 \\
T & G^{-} & \beta U
\end{array}\right)+\left(\begin{array}{ccc}
0 & 0 & 1 \\
z & 0 & 0 \\
0 & z & 0
\end{array}\right),
$$

where $\alpha$ and $\beta$ are arbitrary constant numbers, and $U, G^{ \pm}$and $T$ are the potentials.

Proposition 4.1. From the point of view of our general construction (4.5) is nothing but a possible gauge fixing of the hierarchy corresponding to $\left(\Lambda_{2,0}, w_{c}, \mathbf{s}=(1,0,0)\right)$, for $A_{2}$.

Proof. The proof amounts to showing that (4.5) is a consistent gauge choice. The set $Q$ is in this example

$$
Q=h \cup\left\{E_{-\alpha_{1}-\alpha_{2}}, E_{-\alpha_{1}}, E_{-\alpha_{2}}, E_{\alpha_{1}}, E_{\alpha_{2}}, z E_{-\alpha_{1}-\alpha_{2}}\right\},
$$

whilst $P$, the gauge freedom, is

$$
P=\left\{E_{-\alpha_{1}-\alpha_{2}}, E_{-\alpha_{1}}, E_{-\alpha_{2}}\right\} .
$$

Therefore, we can write the (unifixed) potential as

$$
q=\left(\begin{array}{ccc}
y_{1} & c & 0 \\
e & y_{2} & d \\
a+b z & f & y_{3}
\end{array}\right) .
$$

The gauge transformations which preserve the form of $q$ can be written

$$
q \rightarrow \Phi \partial_{x} \Phi^{-1}+\Phi(q+\Lambda) \Phi^{-1}-\Lambda,
$$


where

$$
\Phi=\left(\begin{array}{lll}
1 & 0 & 0 \\
A & 1 & 0 \\
B & C & 1
\end{array}\right)
$$

Notice that the gauge freedom is exactly the same as for the $A_{2}$-Drinfel'd-Sokolov hierarchy, which is a general feature in the sense that the subspace $P$ only depends on $\mathbf{s}[w]$ and $\mathbf{s}$, and not on the choice of $\Lambda \in \mathscr{H}_{+}[w]$. The proof proceeds by showing that there exists a gauge transformation which brings $q$ into the form

$$
\tilde{q}=\left(\begin{array}{ccc}
(\alpha-\beta) U & 0 & 0 \\
G^{+} & -\alpha U & 0 \\
T & G^{-} & \beta U
\end{array}\right)+\phi\left(\begin{array}{lll}
0 & 1 & 0 \\
0 & 0 & 1 \\
z & 0 & 0
\end{array}\right)
$$

It is not difficult to construct the required gauge transformation, one finds that

$$
\begin{aligned}
& A=\frac{1}{3}(b+c-2 d), \\
& C=\frac{1}{3}(2 c-b-d), \\
& B=-y_{3}-d C-\frac{\beta}{\alpha}\left(A c+y_{2}-d C-C A\right),
\end{aligned}
$$

and $\phi=(b+c+d) / 3$. Having established the form (4.6), we now appeal to Proposition 3.6, (and the discussion following it), which states that the time evolution of the component of $q$ in $\mathscr{H}_{i-1}[w]$ is zero. In our case, $i=2$ and $\hat{g}_{1}\left(\mathbf{s}\left[w_{c}\right]\right)$ is spanned by $\left\{E_{\alpha_{1}}, E_{\alpha_{2}}, z E_{-\alpha_{1}-\alpha_{2}}\right\}$, and there is an element of this vector space which is in $\mathscr{H}_{i-1}[w]$ : it is precisely $\Lambda_{1,0}$, the matrix multiplying $\phi$ in (4.6). The proposition implies that we can consistently impose $\phi=0$, and hence recover the $L$-operator defined in (4.5).

We now construct the first flow of the hierarchy:

$$
\frac{\partial \tilde{L}}{\partial t_{1,0}}=\left[A_{1,0}, \tilde{L}\right]
$$

Proposition 3.8 implies that $A_{1,0}$ has the general form

$$
A_{1,0}=\left(\begin{array}{ccc}
a_{1} & 1 & 0 \\
s & a_{2} & 1 \\
z+v & t & a_{3}
\end{array}\right)
$$

for functions $s, t$ and $a_{i}$, with $\sum_{i=1}^{3} a_{i}=0$. One can then solve the recursion relations that follow from (4.7) to obtain

$$
A_{1,0}=\left(\begin{array}{ccc}
0 & 1 & 0 \\
-(\alpha+\beta) U & 0 & 1 \\
z+\left(1-\frac{\beta}{\alpha}\right) G^{-}+\frac{\beta}{\alpha} G^{+} & (-2 \alpha+\beta) U & 0
\end{array}\right),
$$


and the equations of the flow

$$
\begin{aligned}
\partial_{t_{1,0}} G^{+} & =(\alpha+\beta) U^{\prime}-(\alpha+\beta)(2 \alpha-\beta) U^{2}+T, \\
\partial_{t_{1,0}} G^{-}= & (2 \alpha-\beta) U^{\prime}+(\alpha+\beta)(2 \alpha-\beta) U^{2}-T, \\
\partial_{t_{1}, 0} U= & -\frac{1}{\alpha}\left(G^{-}-G^{+}\right), \\
\partial_{t_{1,0}} T= & -\left(1-\frac{\beta}{\alpha}\right) G^{-\prime}-\frac{\beta}{\alpha} G^{+\prime} \\
& +\frac{2}{\alpha}\left(\alpha^{2}-\alpha \beta+\beta^{2}\right) U\left(G^{-}-G^{+}\right),
\end{aligned}
$$

where prime denotes $\partial_{x}$. To compare with ref. [15] set $\alpha=2 \beta=1$ and put $x \rightarrow-x$.

Notice that the first flow can be written as

$$
\frac{\partial L_{1,0}}{\partial x}=\left[\tilde{q}+\Lambda_{2,0}, L_{1,0}\right]
$$

where $L_{1,0}=\partial_{t_{1,0}}-A$. It is apparent that (4.9) is nothing but the second flow of the $A_{2}$ Drinfel'd-Sokolov KdV hierarchy, with the identifications $x \rightarrow-t_{2,0}$ and $t_{1,0} \rightarrow-x$. In fact, if we choose $\beta+\alpha=0$ then $L_{1,0}$ becomes

$$
L_{1,0}=\partial_{t_{1,0}}-\left(\begin{array}{ccc}
0 & 0 & 0 \\
0 & 0 & 0 \\
2 G^{-}-G^{+} & -3 \alpha U & 0
\end{array}\right)-\left(\begin{array}{ccc}
0 & 1 & 0 \\
0 & 0 & 1 \\
z & 0 & 0
\end{array}\right) \text {. }
$$

This is precisely the conventional form of gauge-fixed $\tilde{L}$-operator for the $A_{2}$-Drinfel'd-Sokolov hierarchy. This leads us to propose the following:

Proposition 4.2. Any solution of $a\left(\Lambda_{i, n}, w, \mathbf{s}\right)$-hierarchy gives a solution of $a\left(\Lambda_{j, m}, w, \mathbf{s}\right)-$ hierarchy, where $\Lambda_{i, n} \in \mathscr{H}_{i+n N}[w], \Lambda_{j, m} \in \mathscr{H}_{j+m N}[w]$. (We are assuming that both hierarchies are of type $I$.)

Proof. The first hierarchy is defined by the zero curvature equations $\left[L_{i, n}, L_{k, p}\right]=$ 0 , where $L_{i, n}=-\tilde{L}$ and the $L_{j, m}$ are defined in (3.18). However, the equations of the hierarchy also implied the more general statement that $\left[L_{k, p}, L_{l, q}\right]=0$. A similar picture emerges for the second hierarchy. The important point to notice is that for both hierarchies the potentials $q_{k, p} \in C^{\infty}\left(\mathbf{R}, Q^{k+p N}\right)$. Furthermore, both the hierarchies have the same gauge invariance. Having a solution of a hierarchy implies that we know $q_{k, p}=q_{k, p}\left(t_{l, q}\right)$. Clearly, a solution of the first hierarchy is also a solution of the second hierarchy, and vice-versa. So the space of solutions of hierarchies can be mapped to each other.

Let us illustrate how this works for the fractional $\mathrm{KdV}$ hierarchy that we are considering. Let us take $\alpha+\beta=0$ and so the first flow has the form (4.10). If we have a solution of the $\mathrm{KdV}$ hierarchy, that is $u_{1}\left(x, t_{2,0}, t_{1,1}, t_{2,1}, t_{1,3}, \ldots\right)$ and $u_{2}\left(x, t_{2,0}, t_{1,1}, t_{2,1}, t_{1,3}, \ldots\right)$ then a solution of the fractional $\mathrm{KdV}$ hierarchy is given relabelling $x \rightarrow-t_{1,0}$ and $t_{2,0} \rightarrow-x$ and the following relations, where for clarity 
we have indicated the dependence only on the relevant variables:

$$
\begin{aligned}
U\left(t_{1,0}, x\right) & =-\frac{1}{3 \alpha} u_{2}\left(-t_{1,0},-x\right), \\
G^{+}\left(t_{1,0}, x\right) & =u_{1}\left(-t_{1,0},-x\right)-\frac{2}{3} \partial_{t_{1,0}} u_{2}\left(-t_{1,0},-x\right), \\
G^{-}\left(t_{1,0}, x\right) & =u_{1}\left(-t_{1,0},-x\right)-\frac{1}{3} \partial_{t_{1,0}} u_{2}\left(-t_{1,0},-x\right), \\
T\left(t_{1,0}, x\right) & =\partial_{t_{1,0}} u_{1}\left(-t_{1,0},-x\right)-\frac{2}{3} \partial_{t_{1,0}}^{2} u_{2}\left(-t_{1,0},-x\right) .
\end{aligned}
$$

Conversely, if we have a solution of the fractional $\mathrm{KdV}$ hierarchy, that is $G^{ \pm}, U$ and $T$ as functions of $\left(t_{1,0}, x, t_{1,1}, t_{2,1}, t_{1,3}, \ldots\right)$, then a solution of the $\mathrm{KdV}$ hierarchy is obtained by relabelling $x \rightarrow-t_{2,0}$ and $t_{1,0} \rightarrow-x$ and

$$
\begin{aligned}
& u_{1}\left(x, t_{2,0}\right)=2 G^{-}\left(-x,-t_{2,0}\right)-G^{+}\left(-x,-t_{2,0}\right), \\
& u_{2}\left(x, t_{2,0}\right)=-3 \alpha U\left(-x,-t_{2,0}\right) .
\end{aligned}
$$

Proposition 4.3. If we call these two maps between the two solution spaces $\mathscr{F}_{1}: \mathrm{KdV} \rightarrow \mathrm{FKdV}$ and $\mathscr{F}_{2}: \mathrm{FKdV} \rightarrow \mathrm{KdV}$, then $\mathscr{F}_{1} \circ \mathscr{F}_{2}$ and $\mathscr{F}_{2} \circ \mathscr{F}_{1}$ are both the identity.

The proof of this is straightforward, and uses the equations of the first flow (4.8). In summary, the following picture has emerged. For any choice of $w$ and $\mathbf{s}$ one can define a sequence of hierarchies by choosing for $\Lambda$ each (regular) element of the Heisenberg subalgebra $\mathscr{H}_{+}[w]$. Each of these hierarchies has the same gauge invariance and after gauge fixing the solution spaces of each hierarchy are isomorphic. The only difference between the hierarchies is in the identification of the defining variable $x$; in this sense there is a complete democracy between the flows. One might expect, therefore, that these hierarchies will lead to different Hamiltonian structures as claimed in [15] for $A_{2}$.

4.3. Homogeneous Hierarchies. In this section we consider hierarchies for which $w=1$, the identity element of $W_{g}$. So in this case $\mathbf{s}[\mathbf{1}]$ is the homogeneous gradation. For these hierarchies there is only an analogue of the $\mathrm{mKdV}$ hierarchy, since there is no lower gradation in the partial ordering. The simplest such type I hierarchy has

$$
L=\partial_{x}+q+\Lambda
$$

where $\Lambda=z \mu \cdot H$, for some $\mu$. In order that the hierarchy be of type I we require that $\mu \cdot H$ be regular, and so $\alpha \cdot \mu \neq 0 \forall \alpha \in \Phi_{g}$, the root system of $g$. Following the general construction of Sect. $3.1 q \in C^{\infty}(\mathbf{R}, g)$. In components

$$
q=f \cdot H+\sum_{\alpha \in \Phi_{g}} q^{\alpha} E_{\alpha}
$$

for functions $f$ and $q^{\alpha}$. Using the arguments following Proposition 3.6 we see that $f$ is constant along the flows, and so we set it to zero.

The Heisenberg algebra $\mathscr{H}[1]$ is spanned by the elements $z^{n} v \cdot H$, and so at each level there is a vector space, of dimension $r$, of elements of $\mathscr{H}$ [1]. Therefore, at each level we can define a vector space of flows. For example the flows with $n=1$ are given by

$$
\frac{\partial L}{\partial t_{v, 1}}=\left[a_{0}+z v \cdot H, L\right]
$$


which gives the following equation for $a_{0}$ :

and the flow

$$
[v \cdot H, q]+\left[a_{0}, \mu \cdot H\right]=0,
$$

$$
\frac{\partial q}{\partial t_{v, 1}}=-\partial_{x} a_{0}+\left[a_{0}, q\right]
$$

Solving (4.11) we find

$$
a_{0}=\sum_{\alpha \in \Phi_{g}}\left(\frac{v \cdot \alpha}{\mu \cdot \alpha}\right) q^{\alpha} E_{\alpha},
$$

and so the first vector space of flows is

$$
\frac{\partial q^{\alpha}}{\partial t_{v, 1}}=v \cdot\left(-\frac{\alpha}{(\mu \cdot \alpha)} \partial_{x} q^{\alpha}+\sum_{\beta+\gamma=\alpha} \frac{\beta}{(\mu \cdot \beta)} N_{\beta, \gamma} q^{\beta} q^{\gamma}\right),
$$

where $N_{\beta, \gamma}$, for $\beta+\gamma \in \Phi_{g}$, are the structure constants of $g$.

The second vector space of flows is

$$
\begin{aligned}
\frac{\partial q^{\alpha}}{\partial t_{v, 2}}= & v \cdot\left(\frac{\alpha}{(\mu \cdot \alpha)^{2}} \partial_{x}^{2} q^{\alpha}-\frac{1}{\mu \cdot \alpha} \sum_{\beta+\gamma=\alpha} \frac{\beta}{(\mu \cdot \beta)} N_{\beta, \gamma} \partial_{x}\left(q^{\beta} q^{\gamma}\right)\right. \\
& -\frac{1}{2} \sum_{\beta} \frac{\beta(\alpha \cdot \beta)}{(\mu \cdot \beta)^{2}} q^{\beta} q^{-\beta} q^{\alpha}-\sum_{\beta+\gamma=\alpha} \frac{\beta}{(\mu \cdot \beta)^{2}} N_{\beta, \gamma}\left(\partial_{x} q^{\beta}\right) q^{\gamma} \\
& \left.+\sum_{\substack{\beta+\gamma=\alpha \\
\beta^{\prime}+\gamma^{\prime}=\beta}} \frac{\beta^{\prime}}{(\mu \cdot \beta)\left(\mu \cdot \beta^{\prime}\right)} N_{\beta, \gamma} N_{\beta^{\prime}, \gamma^{\prime}} q^{\beta^{\prime}} q^{\gamma^{\prime}} q^{\gamma}\right) .
\end{aligned}
$$

When $g=A_{1}$, the construction above is the non-linear Schrödinger hierarchy, associated to the $L$ operator

$$
L=\partial_{x}+\left(\begin{array}{ll}
0 & \psi \\
\bar{\psi} & 0
\end{array}\right)+z\left(\begin{array}{ll}
1 & 0 \\
0 & 1
\end{array}\right)
$$

So it is natural to view the homogeneous hierarchies as generalization of the nonlinear Schrödinger hierarchy. We have only considered the case where $\Lambda$ is regular, i.e. type I hierarchies. In general there are many other possibilities [16].

4.4. Hierarchy Associated to $w=R_{\alpha_{1}}$ in $A_{2}$. The Weyl group of $A_{2}$ has three conjugacy classes corresponding to the following Carter diagrams: $A_{2}$, corresponding to the Coxeter element, $A_{1}$, corresponding to the conjugacy class $\left\{R_{\alpha_{1}}, R_{\alpha_{2}}\right.$, $\left.R_{\alpha_{1}} R_{\alpha_{2}} R_{\alpha_{1}}\right\}$, and $\varnothing$, corresponding to the identity. We have already dealt with the hierarchies that can be constructed from the Coxeter element, which leads to the conventional generalized $\mathrm{KdV}$ and its fractional generalizations, and the identity element, which leads to the "homogeneous hierarchy" of Sect. 4.3. In this section we consider the hierarchies associated to the third conjugacy class.

We take $w=R_{\alpha_{1}} R_{\alpha_{2}} R_{\alpha_{1}}$ as the representative of the conjugacy class this corresponds to the Weyl reflection in the root $\alpha_{0}=-\alpha_{1}-\alpha_{2}$, under which:

$$
\begin{aligned}
& \alpha_{1} \mapsto-\alpha_{2} \\
& \alpha_{2} \mapsto-\alpha_{1} .
\end{aligned}
$$


Obviously, $w$ has order $2, w^{2}=1$. The lift $\hat{w}: A_{2} \rightarrow A_{2}$ such that

has the property

$$
H^{\prime} \mapsto w\left(H^{\prime}\right), \quad E_{\alpha}^{\prime} \mapsto \psi_{\alpha} E_{w(\alpha)}^{\prime},
$$

$$
\hat{w}^{2}\left(E_{\alpha}^{\prime}\right)=(-1)^{\tilde{\alpha} \cdot \alpha} E_{\alpha}^{\prime}, \quad \tilde{\alpha}=\alpha+w(\alpha),
$$

so the order doubles and become $4\left(\hat{w}^{2}\left(E_{\alpha_{1,2}}^{\prime}\right)=-E_{\alpha_{1,2}}^{\prime}\right)$. The result follows because $A_{2}$ is simply-laced (see the remark following Theorem 2.3).

Since $\hat{w}$ is an inner automorphism of $A_{2}$, we may realize it in the "shifted picture" of (2.1). In this basis,

$$
\hat{w}: H \mapsto H, \quad E_{\alpha} \mapsto e^{2 \pi i \alpha \cdot \delta} E_{\alpha},
$$

where the shift vector, in this case, is $\delta=\frac{1}{4}\left(\omega_{1}+\omega_{2}\right)\left(\left(s_{i}\right)=(2,1,1)\right)$. In this basis, $A_{2}$ decomposes into the following eigenspaces under $\hat{w}, \bigoplus_{i} g_{i}$ :

$$
\begin{gathered}
g_{0}=\left\{H_{1}, H_{2}\right\} ; \quad g_{1}=\left\{E_{\alpha_{1}}, E_{\alpha_{2}}\right\} ; \quad g_{2}=\left\{E_{\alpha_{1}+\alpha_{2}}\right\} \\
g_{-1}=\left\{E_{-\alpha_{1}}, E_{-\alpha_{2}}\right\} ; \quad g_{-2}=\left\{E_{-\alpha_{1}-\alpha_{2}}\right\},
\end{gathered}
$$

which defines a new gradation of the loop algebra $\hat{A}_{2}$ given by the derivation $d_{\mathrm{s}[w]}$, of Definition 2.1:

$$
\left[d_{\mathrm{s}[w]}, a \otimes z^{n}\right]=(i+4 n)\left(a \otimes z^{n}\right), \quad a \in g_{i} .
$$

The Heisenberg subalgebra, $\mathscr{H}[w]$, corresponding to this element of the Weyl group is

$$
\begin{aligned}
\mathscr{H}[w] & =\bigoplus_{n \in \mathbf{Z}}\left(\left(\alpha_{1}-\alpha_{2}\right) \cdot H \otimes z^{n}\right) \bigoplus_{m \in \mathbf{Z}}\left(\left(E_{\alpha_{1}+\alpha_{2}} \otimes 1+E_{-\alpha_{1}-\alpha_{2}} \otimes z\right) \otimes z^{m}\right) \\
& \in \bigoplus_{n \in \mathbf{Z}} \hat{g}_{4 n}(\mathbf{s}[w]) \bigoplus_{m \in \mathbf{Z}} \hat{g}_{2+4 m}(\mathbf{s}[w]),
\end{aligned}
$$

Obviously, we can write

$$
\mathscr{H}[w]=\bigoplus_{\substack{j \in I[w] \\ n \in \mathbf{Z}}}\left(h_{j} \otimes z^{n}\right)
$$

where for this element of the Weyl group $I[w]=\{0,2\}$, and

$$
\begin{aligned}
& h_{0}=\left(\alpha_{1}-\alpha_{2}\right) \cdot H=\left(\begin{array}{ccc}
1 & 0 & 0 \\
0 & -2 & 0 \\
0 & 0 & 1
\end{array}\right) \\
& h_{2}=E_{\alpha_{1}+\alpha_{2}} \otimes 1+E_{-\alpha_{1}-\alpha_{2}} \otimes z=\left(\begin{array}{lll}
0 & 0 & 1 \\
0 & 0 & 0 \\
z & 0 & 0
\end{array}\right),
\end{aligned}
$$

in the three-dimensional representation of $A_{2}$.

Let us now construct the generalized hierarchies associated to $w$. We define the operator $L$ in the space $C^{\infty}\left(\mathbf{R}, \hat{A}_{2}\right)$

$$
L=\partial_{x}+\Lambda+q
$$

$\Lambda$ is a constant "regular" element of $\mathscr{H}_{+}[w]$, and so the hierarchy is of type $\mathrm{I}$. 
We shall take $\Lambda=h_{2}$, which is obviously regular. Given some other gradation of $\hat{A}_{2}, \mathbf{s} \leqq \mathbf{s}[w]$, the potential $q$ is an element of the following space:

$$
q \in C^{\infty}(\mathbf{R}, Q), \quad Q=\hat{g}_{\geqq 0}(\mathbf{s}) \cap \hat{g}_{<i}(\mathbf{s}[w]) .
$$

Such a operator is invariant under gauge transformations $L^{\prime}=\exp (\operatorname{ad} S)(L)$, with

$$
S \in C^{\infty}(\mathbf{R}, P), \quad P=\hat{g}_{\geqq 0}(\mathbf{s}) \cap \hat{g}_{<0}(\mathbf{s}[w]) .
$$

If we choose $\mathrm{s}$ to be the homogeneous gradation, then the hierarchy has maximal gauge invariance, and

$$
q=\left(\begin{array}{ccc}
* & * & 0 \\
* & * & * \\
* & * & *
\end{array}\right), \quad S=\left(\begin{array}{lll}
0 & 0 & 0 \\
* & 0 & 0 \\
* & * & 0
\end{array}\right)
$$

Conversely, if we choose $\mathbf{s}$ to be the same as $\mathbf{s}[w]$, then the hierarchy has no gauge freedom at all and the potential is

$$
q=q_{0}+q_{1}=\left(\begin{array}{ccc}
u^{(+)}+u^{(-)} & q^{(1)} & 0 \\
0 & -2 u^{(-)} & q^{(2)} \\
0 & 0 & -u^{(+)}+u^{(-)}
\end{array}\right),
$$

where $u^{( \pm)}$parametrize $q_{0}$, and $q^{(1,2)}$ parametrize $q_{1}$. Let us work out this (gauge fixed) case, which leads to a generalized modified hierarchy.

Following the steps of Sect. 3.1, first we have to "diagonalize" $L$

$$
\exp (\operatorname{ad} T)(L)=L_{0}=\partial_{x}+h_{2}+\sum_{j=0}^{\infty} H_{-2 j} .
$$

Proposition 3.6 implies that the $H_{j} \in C^{\infty}\left(\mathbf{R}, \mathscr{H}_{j}[w]\right)$ will be the conserved densities of this hierarchy. In the matrix representation we are using,

$$
L_{0}=\Phi L \Phi^{-1}, \quad \Phi=1+\sum_{j=1}^{\infty} \Phi_{-j}
$$

and we obtain a series of recursion relations

$$
\begin{aligned}
& {\left[\Lambda, \Phi_{-1}\right]+H_{1}=q_{1},} \\
& {\left[\Lambda, \Phi_{-2}\right]+H_{0}=q_{0}+\Phi_{-1} q_{1}-H_{1} \Phi_{-1},} \\
& {\left[\Lambda, \Phi_{-j-2}\right]+H_{-j}=\Phi_{-j} q_{0}+\Phi_{-j-1} q_{1}-\Phi_{-j}^{\prime}-\sum_{k=1}^{j+1} H_{k-j} \Phi_{-k}, j=1, \ldots, \infty,}
\end{aligned}
$$

from which the $H_{j}$ 's and the $\Phi_{-j}$ 's, up to pieces in $\operatorname{Ker}(\operatorname{ad} \Lambda)$, can be obtained. Given that $\mathscr{H}(\mathbf{s}[w])$ lives in a subspace of $\hat{g}$ of even grade, the first equation implies that $q_{1} \in \operatorname{Im}(\operatorname{ad} \Lambda)$, which is already the case. Solving for the first few terms

$$
\begin{aligned}
\Phi_{-1} & =q^{(1)} E_{-\alpha_{2}}-q^{(2)} E_{-\alpha_{1}}, \\
\Phi_{-2} & =\frac{1}{2}\left(u^{(+)}-\frac{1}{2} q^{(1)} q^{(2)}\right) \hat{F}, \\
H_{0} & =\left(u^{(-)}+\frac{1}{2} q^{(1)} q^{(2)}\right) h_{0},
\end{aligned}
$$




$$
\begin{aligned}
\Phi_{-3}= & {\left[-\partial_{x} q^{(1)}+\left(\frac{1}{2} u^{(+)}-3 u^{(-)}-\frac{3}{4} q^{(1)} q^{(2)}\right) q^{(1)}\right] E_{\alpha_{1}} \otimes z^{-1} } \\
& +\left[-\partial_{x} q^{(2)}+\left(u^{(+)}+3 u^{(-)}+q^{(1)} q^{(2)}\right) q^{(2)}\right] E_{\alpha_{2}} \otimes z^{-1} \\
\Phi_{-4}= & \frac{1}{4}\left[q^{(2)} \partial_{x} q^{(1)}+\frac{1}{2} \partial_{x}\left(q^{(1)} q^{(2)}\right)-\partial_{x} u^{(+)}-u^{(+)} q^{(1)} q^{(2)}+3 u^{(-)} q^{(1)} q^{(2)}\right. \\
& \left.+\left(q^{(1)} q^{(2)}\right)^{2}\right]\left(h_{+} \otimes z^{-1}\right), \\
H_{-2}= & {\left[-\frac{1}{2} q^{(2)} \partial_{x} q^{(1)}+\frac{1}{2}\left(u^{(+)}\right)^{2}-\frac{3}{2} q^{(1)} q^{(2)} u^{(-)}-\frac{3}{8}\left(q^{(1)} q^{(2)}\right)^{2}\right] h_{2} \otimes z^{-1}, }
\end{aligned}
$$

where

$$
h_{+}=\left(\begin{array}{ccc}
1 & 0 & 0 \\
0 & 0 & 0 \\
0 & 0 & -1
\end{array}\right), \quad \hat{F}=\left(\begin{array}{ccc}
0 & 0 & -z^{-1} \\
0 & 0 & 0 \\
1 & 0 & 0
\end{array}\right) \text {. }
$$

Once $\Phi$ has been obtained, the flows are found from (3.8),

$$
\frac{\partial L}{\partial t_{i, n}}=\left[\left(\Phi^{-1} b \Phi\right)_{+}, L\right],
$$

for each $b \in \mathscr{H}_{i+4 n}[w]$, with $i=0,2$ and $i+4 n>0$. The first one, $(2,0)$, corresponds to $b=h_{2} \in \mathscr{H}_{2}[w]$,

$$
\begin{aligned}
& \frac{\partial u^{(+)}}{\partial t_{2,0}}=-\partial_{x} u^{(+)} \\
& \frac{\partial u^{(-)}}{\partial t_{2,0}}=\frac{1}{2} \partial_{x}\left(q^{(1)} q^{(2)}\right) \\
& \frac{\partial q^{(1)}}{\partial t_{2,0}}=-\partial_{x} q^{(1)}-3\left(u^{(-)}+\frac{1}{2} q^{(1)} q^{(2)}\right) q^{(1)} \\
& \frac{\partial q^{(2)}}{\partial t_{2,0}}=-\partial_{x} q^{(2)}+3\left(u^{(-)}+\frac{1}{2} q^{(1)} q^{(2)}\right) q^{(2)}
\end{aligned}
$$

This flow implies that the conserved density $H_{0}$ is, in fact, a constant,

$$
\frac{\partial H_{0}}{\partial t_{2,0}}=\frac{\partial\left(u^{(-)}+\frac{1}{2} q^{(1)} q^{(2)}\right)}{\partial t_{2,0}}=0 .
$$

Notice that this is just a consequence of Proposition 3.6. So we can put $H_{0}=0$, which reduces by one the number of independent potentials (degrees of freedom) and then the flow simply identifies $x$ and $t_{2,0}$ :

$$
\begin{array}{ll}
\frac{\partial u^{(+)}}{\partial t_{2,0}}=-\partial_{x} u^{(+)}, & \frac{\partial u^{(-)}}{\partial t_{2,0}}=-\partial_{x} u^{(-)} \\
\frac{\partial q^{(1)}}{\partial t_{2,0}}=-\partial_{x} q^{(1)}, & \frac{\partial q^{(2)}}{\partial t_{2,0}}=-\partial_{x} q^{(2)}
\end{array}
$$

Of course, one is redundant.

The second flow, $(0,1)$, corresponds to $b=h_{0} \otimes z \in \mathscr{H}_{4} . H_{0}$ is also constant 
under this flow.

$$
\begin{aligned}
\frac{\partial u^{(+)}}{\partial t_{0,1}}= & -\frac{3}{2} \partial_{x}\left(\partial_{x}\left(q^{(1)} q^{(2)}\right)-2 u^{(+)} q^{(1)} q^{(2)}\right) \\
\frac{\partial u^{(-)}}{\partial t_{0,1}}= & -\frac{3}{2} \partial_{x}\left(q^{(2)} \partial_{x} q^{(1)}-q^{(1)} \partial_{x} q^{(2)}-\left(q^{(1)} q^{(2)}\right)^{2}\right) \\
\frac{\partial q^{(1)}}{\partial t_{0,1}}= & 3 \partial_{x}\left(\partial_{x} q^{(1)}-\left(u^{(+)}+\frac{1}{2} q^{(1)} q^{(2)}\right) q^{(1)}\right) \\
& +3\left(\left(u^{(+)}+\frac{1}{2} q^{(1)} q^{(2)}\right) \partial_{x} q^{(1)}-\left(q^{(1)}\right)^{2} \partial_{x} q^{(2)}-\left(\left(u^{(+)}\right)^{2}+\frac{3}{4}\left(q^{(1)} q^{(2)}\right)^{2}\right) q^{(1)}\right) \\
\frac{\partial q^{(2)}}{\partial t_{0,1}}= & -3 \partial_{x}\left(\partial_{x} q^{(2)}-\left(u^{(+)}-\frac{1}{2} q^{(1)} q^{(2)}\right) q^{(2)}\right) \\
& -3\left(\left(u^{(+)}-\frac{1}{2} q^{(1)} q^{(2)}\right) \partial_{x} q^{(2)}+\left(q^{(2)}\right)^{2} \partial_{x} q^{(1)}-\left(\left(u^{(+)}\right)^{2}+\frac{3}{4}\left(q^{(1)} q^{(2)}\right)^{2}\right) q^{(2)}\right)
\end{aligned}
$$

The first non-trivial conserved density is

$$
H_{-2}=-\frac{1}{2} q^{(2)} \partial_{x} q^{(1)}+\frac{1}{2}\left(u^{(+)}\right)^{2}+\frac{3}{8}\left(q^{(1)} q^{(2)}\right)^{2} .
$$

\section{Discussion and Conclusions}

In the preceding sections we have described a way of generalizing the approach adopted by Drinfel'd and Sokolov towards constructing integrable hierarchies of partial differential equations. The main protagonists were Kac-Moody algebras and their maximal Heisenberg subalgebras. Although for simplicity's sake we only considered the untwisted Kac-Moody algebras, there is no reason why similar constructions might not be undertaken with the twisted Kac-Moody algebras (Drinfel'd and Sokolov considered both twisted and untwisted algebras).

Our main result was that for each positive element of the Heisenberg subalgebra of an (untwisted) Kac-Moody algebra, we could define a collection of hierarchies consisting of generalized KdV's, a modified $\mathrm{KdV}$, and various partially modified KdV's. The various hierarchies in the collection for fixed $\Lambda$ are related by generalized Miura maps. So up to the equivalence under Miura maps, the inequivalent hierarchies are given by the inequivalent Heisenberg subalgebras of the Kac-Moody algebra. These, in turn, are in one-to-one correspondence with conjugacy classes of the Weyl group of the finite Lie algebra. So loosely speaking, there is a hierarchy for each conjugacy class of the Weyl group.

The so-called "fractional" $\mathrm{KdV}$ hierarchies of [15] are naturally contained in our framework, and result from taking $\Lambda$ to be an element of $\mathscr{H}_{+}[w]$ of arbitrary grade. We showed that the solution spaces of such hierarchies are identical, differing only in the choice of the variable $x$ from amongst the flow variables. It is well-known that the modified $\mathrm{KdV}$ hierarchies of Drinfel'd and Sokolov admit a single local Hamiltonian structure, whereas their $\mathrm{KdV}$ hierarchies admit two distinct Hamiltonian structures, a fact that has been known for some time for the original $\mathrm{KdV}$ hierarchy. 
The second Hamiltonian structure of the Drinfel'd-Sokolov KdV hierarchies are the classical $W$-algebras, so it is natural to ask whether more general classical $W$-algebras appear from the hierarchies introduced above, as it appears they do [15]. There is also the question of whether the different hierarchies corresponding to different Weyl conjugacy classes, can be written in a Hamiltonian form. We will address these questions elsewhere.

It is an interesting issue as to whether the hierarchies that we have constructed are related to the "tau-function" approach to integrable structures. In [10], to each Heisenberg subalgebra of a simply-laced untwisted Kac-Moody algebra, a realization of the basic representations (level 1) were found. Using each of these "vertex operator" representations, Kac and Wakimoto [17] have constructed an integrable hierarchy. It is natural to ask whether these hierarchies are related to the ones that have been considered here. Indeed in the formalism of [17], the usual Drinfel'd-Sokolov $\mathrm{KdV}$ is obtained from the vertex construction associated to the Coxeter element of $W_{g}$. For more general conjugacy classes of $W_{g}$ it is not known whether the two constructions lead to the same hierarchy. For the case of the $\mathrm{KdV}$ hierarchy, where $g=A_{1}$, a direct connexion between the tau-function and zero-curvature formalisms has been established by Wilson [18], employing the dressing procedure [14]. It would be interesting to try and extend this work to the more general hierarchies.

Acknowledgement. We would like to thank Nigel Burroughs for many discussions on the topics considered in this paper, and Jan Lacki for explaining his recent work. The research of JLM is supported by a Fullbright/MEC fellowship, that of MFdeG by the Natural Sciences and Engineering Research Council of Canada and that of TJH by NSF PHY90-21984.

\section{References}

1. Newell, A.: Solitons in mathematics and physics. Philadelphia. Society for Industrial and Applied Mathematics 1985

2. Drinfel'd, V. G., Sokolov, V. V.: Lie algebras and equations of the Korteweg-de Vries Type. J. Sov. Math. 30 (1985) 1975; Equations of Korteweg-De Vries type and simple Lie algebras. Sov. Math. Dokl. 23, 457 (1981)

3. Wilson, G. W.: The modified Lax and two-dimensional Toda Lattice equations associated with simple Lie Algebras. Ergod. Theoret. Dyn. Syst. 1, 361 (1981)

4. Douglas, M.: Strings in less than one-dimension and the generalized $\mathrm{KdV}$ hierarchies. Phys. Lett. B238, 176 (1990)

5. Kazama, Y., Suzuki, H.: Characterization of $N=2$ superconformal models generated by Coset space method. Phys. Lett. 216B, 112 (1989); New $N=2$ superconformal field theories and superstring compactification. Nucl. Phys. B321, 232 (1989)

6. Polyakov, A. M.: Quantum gravity in two-dimensions. Mod. Phys. Lett. A2, 893 (1987); Knizhnik, V. G., Polyakov, A. M., Zamalodchikov, A. B.: Fractal structure of 2d quantum gravity. Mod. Phys. Lett. A3, 819 (1988); Polyakov, M. A.: Gauge transformations and diffeomorphisms. Int. J. Mod. Phys. A5, 833 (1990)

7. Bershadsky, M.: Conformal field theories via Hamiltonian reduction, IAS preprint, IASSNSHEP-90/44; Polyakov, A.: Gauge transformations and diffeomorphisms. Int. J. Mod. Phys. A5, 833 (1990)

8. Kac, V. G.: Infinite dimensional Lie algebras, $2^{\text {nd }}$ ed. Cambridge: Cambridge University Press, 1985

9. Kac, V. G.: Automorphisms of finite order of semi-simple Lie algebras. Funct. Anal. Its Appl. 3, 252 (1969) 
10. Kac, V. G., Peterson, D. H.: 112 Constructions of the basic representation of the loop group of $E_{8}$. In: Symposium on anomalies, geometry and topology. Bardeen, W. A., White, A. R. (eds). Singapore: World Scientific 1985

11. Carter, R. W.: Conjugacy classes in the Weyl group. Composito Mathematica. 25, Fac.1. 1 (1972)

12. Hollowood, T. J., Myhill, R. G.: The 112 Breakings of $E_{8}$. Int. J. Mod. Phys. A3, 899 (1988)

13. Myhill, R. G.: Automorphisms and twisted vertex operators. Ph.D. Thesis, Durham 1987

14. Zakharov, V. E., Shabat, A. B.: A Scheme of Integrating Nonlinear Equations of Mathematical Physics by the Method of the Inverse Scattering Problem I. Funkts. Anal. Pril. 8, 54 (1974); Integration of Nonlinear Equations of Mathematical Physics by the Method of the Inverse Scattering Problem II. Funkts. Anal. Pril. 13, 13 (1979)

15. Bakas, I., Depireux, D. A.: The origins of gauge symmetries in integrable systems of KdV Type. Univ. of Maryland preprint, UMD-PP91-111 (1990); Bakas, I., Depireux, D. A.: A Fractional KdV Hierarchy, Univ. of Maryland preprint, UMD-PP91-168 (1990)

16. Fordy, A. P.: Generalized derivative nonlinear Schrödinger equations and Hermitian symmetric spaces. J. Phys. A17, 1235 (1984)

17. Kac, V. G., Wakimoto, M.: Exceptional hierarchies of soliton equations. Proceed. Symp. Pure Math. 49, 191 (1989)

18. Wilson, G. W.: Infinite-dimensional Lie groups and algebraic geometry in soliton theory. Phil. Trans. R. Soc. Lond. A315, 393 (1985)

Communicated by A. Jaffe 